\title{
ON LIPSCHITZ CONTINUITY OF SOLUTIONS OF HYPERBOLIC POISSON'S EQUATION
}

\author{
JIAOLONG CHEN, MANZI HUANG, ANTTI RASILA, AND XIANTAO WANG *
}

\begin{abstract}
In this paper, we investigate solutions of the hyperbolic Poisson equation $\Delta_{h} u(x)=\psi(x)$, where $\psi \in L^{\infty}\left(\mathbb{B}^{n}, \mathbb{R}^{n}\right)$ and

$$
\Delta_{h} u(x)=\left(1-|x|^{2}\right)^{2} \Delta u(x)+2(n-2)\left(1-|x|^{2}\right) \sum_{i=1}^{n} x_{i} \frac{\partial u}{\partial x_{i}}(x)
$$

is the hyperbolic Laplace operator in the $n$-dimensional space $\mathbb{R}^{n}$ for $n \geq 2$. We show that if $n \geq 3$ and $u \in C^{2}\left(\mathbb{B}^{n}, \mathbb{R}^{n}\right) \cap C\left(\overline{\mathbb{B}^{n}}, \mathbb{R}^{n}\right)$ is a solution to the hyperbolic Poisson equation, then it has the representation $u=P_{h}[\phi]-G_{h}[\psi]$ provided that $\left.u\right|_{\mathbb{S}^{n-1}}=\phi$ and $\int_{\mathbb{B}^{n}}\left(1-|x|^{2}\right)^{n-1}|\psi(x)| d \tau(x)<\infty$. Here $P_{h}$ and $G_{h}$ denote Poisson and Green integrals with respect to $\Delta_{h}$, respectively. Furthermore, we prove that functions of the form $u=P_{h}[\phi]-G_{h}[\psi]$ are Lipschitz continuous.
\end{abstract}

\section{INTRODUCTION AND MAIN RESUlTS}

For $n \geq 2$, let $\mathbb{B}^{n}\left(x_{0}, r\right)=\left\{x \in \mathbb{R}^{n}:\left|x-x_{0}\right|<r\right\}, \overline{\mathbb{B}^{n}}\left(x_{0}, r\right)=\left\{x \in \mathbb{R}^{n}:\left|x-x_{0}\right| \leq\right.$ $r\}$ and $\mathbb{S}^{n-1}\left(x_{0}, r\right)=\partial \mathbb{B}^{n}\left(x_{0}, r\right)$. We write $\mathbb{B}^{n}=\mathbb{B}^{n}(0,1)$ and $\mathbb{S}^{n-1}=\mathbb{S}^{n-1}(0,1)$.

Let $L_{1}, L_{2}$ be two constants and $\Omega \subset \mathbb{R}^{n}$ a domain. Then a mapping $f: \Omega \rightarrow \mathbb{R}^{n}$ is said to be $L_{1}$-Lipschitz if $|f(x)-f(y)| \leq L_{1}|x-y|$ for all $x, y \in \Omega$, and $L_{2}$-coLipschitz if $|f(x)-f(y)| \geq L_{2}|x-y|$ for all $x, y \in \Omega$. If $f$ is both $L_{1}$-Lipschitz and $L_{2}$-co-Lipschitz for constants $L_{1}$ and $L_{2}$, then $f$ is called bi-Lipschitz.

In [22], Kalaj and Pavlović studied the bi-Lipschitz continuity of quasiconformal self-mappings of the unit disk $\mathbb{D}=\mathbb{B}^{2}$ satisfying the Poisson's equation $\Delta u=\psi$, where $\Delta$ is the usual Laplacian in $\mathbb{R}^{n}$. See $[7,8,17,19,20,21,25,26]$ and references therein for further discussions along this line in the plane.

In [3], Arsenović et al. showed that the Lipschitz continuity of $\phi: \mathbb{S}^{n-1} \rightarrow \mathbb{R}^{n}$ implies the Lipschitz continuity of its harmonic extension $P[\phi]: \mathbb{B}^{n} \rightarrow \mathbb{R}^{n}$ provided that $P[\phi]$ is a $K$-quasiregular mapping. Here $P$ is the usual Poisson kernel with respect to $\Delta$, i.e.

$$
P[\phi](\eta)=\int_{\mathbb{S}^{n-1}} P(\eta, \xi) \phi(\xi) d \sigma(\xi) \text { and } P(\eta, \xi)=\frac{1-|\eta|^{2}}{|\eta-\xi|^{n}}
$$

where $\eta \in \mathbb{B}^{n}$ and $\sigma$ is the $(n-1)$-dimensional Lebesgue measure normalized so that $\sigma\left(\mathbb{S}^{n-1}\right)=1$. Moreover, Kalaj [16] also proved the Lipschitz continuity of

2000 Mathematics Subject Classification. Primary: 31B05; Secondary: 31C05.

Key words and phrases. Lipschitz continuity, hyperbolic Poisson's equation, Poisson kernel, Green function.

*Corresponding author.

The research was partly supported by NSF of China (No. 11571216). 
$P[\phi]: \mathbb{B}^{n} \rightarrow \mathbb{B}^{n}$ under an additional assumption that it is a $K$-quasiconformal harmonic mapping with $P[\phi](0)=0$ and $\phi \in C^{1, \alpha}$ for some $\alpha \in(0,1]$. Later, in [18], Kalaj proved that $K$-quasiconformal mappings of $\mathbb{B}^{n}$ onto itself are Lipschitz, provided that they satisfy the Poisson equation $\Delta u=\psi$ with $\psi \in L^{\infty}\left(\overline{\mathbb{B}^{n}}, \mathbb{R}^{n}\right)$ and $u(0)=0$.

1.1. Main results. The purpose of this paper is to consider results of the above type for solutions of the hyperbolic Laplace equation.

Definition 1.1. A function $u \in C^{2}\left(\mathbb{B}^{n}, \mathbb{R}^{n}\right)(n \geq 2)$ is said to be hyperbolic harmonic $[30,34,35,36]$ if it satisfies the hyperbolic Laplace equation

$$
\Delta_{h} u(x)=\left(1-|x|^{2}\right)^{2} \Delta u(x)+2(n-2)\left(1-|x|^{2}\right) \sum_{i=1}^{n} x_{i} \frac{\partial u}{\partial x_{i}}(x)=0 .
$$

Obviously, for $n=2$, hyperbolic harmonic functions coincide with harmonic functions. See $[10,11]$ for the properties of harmonic mappings. Also, see $\S 2.6$ below for more properties of $\Delta_{h}$.

It is well known that if $u$ satisfies the conditions: (1) $\Delta u=\psi$ which is continuous in $\mathbb{B}^{n}$ with $n \geq 2$, and (2) $\left.u\right|_{\mathbb{S}^{n-1}}=\phi$ which is bounded and integrable in $\mathbb{S}^{n-1}$, then (cf. [15, p. 118-119] or $[18,22,23]$ )

$$
u=P[\phi]-G[\psi] \text { and } G[\psi](\eta)=\int_{\mathbb{B}^{n}} G(\eta, \xi) \psi(\xi) d V(\xi),
$$

where $V$ is the $n$-dimensional Lebesgue volume measure and $G(\eta, \xi), \eta \neq \xi$, is the usual Green function [18, 22, 23], i.e.

$$
G(\eta, \xi)=\left\{\begin{aligned}
\frac{1}{2 \pi} \log \left|\frac{1-\eta \bar{\xi}}{\eta-\xi}\right|, & \text { for } n=2, \\
\frac{1}{(n-2) \omega_{n-1}}\left(|\eta-\xi|^{2-n}-|\xi| \eta|-\eta /| \eta||^{2-n}\right), & \text { for } n \geq 3 .
\end{aligned}\right.
$$

Here $\omega_{n-1}=2 \pi^{n / 2} / \Gamma(n / 2)$ is the $(n-1)$-dimensional surface area of $\mathbb{S}^{n-1}$ and $\Gamma$ is the Gamma function (see e.g. [2, p. 61] or [5, Appendix A]).

The first aim of this paper is to establish the counterpart of the above result to the solutions to the Dirichlet problem:

$$
\left\{\begin{aligned}
\Delta_{h} u(x)=\psi(x), & x \in \mathbb{B}^{n} \\
u(\xi)=\phi(\xi), & \xi \in \mathbb{S}^{n-1},
\end{aligned}\right.
$$

where $\psi \in L^{\infty}\left(\mathbb{B}^{n}, \mathbb{R}^{n}\right)$ and $\phi \in L^{\infty}\left(\mathbb{S}^{n-1}, \mathbb{R}^{n}\right)$.

Denote by $\tau$ the Möbius invariant measure in $\mathbb{B}^{n}$, which is given by

$$
d \tau(x)=\frac{d \nu(x)}{\left(1-|x|^{2}\right)^{n}},
$$

where $\nu$ is the $n$-dimensional Lebesgue volume measure normalized so that $\nu\left(\mathbb{B}^{n}\right)=$ 1. Our result is as follows:

Theorem 1.1. Suppose $u \in C^{2}\left(\mathbb{B}^{n}, \mathbb{R}^{n}\right) \cap C\left(\overline{\mathbb{B}^{n}}, \mathbb{R}^{n}\right), n \geq 3$ and

$$
\int_{\mathbb{B}^{n}}\left(1-|x|^{2}\right)^{n-1}|\psi(x)| d \tau(x) \leq \mu_{1}
$$


where $\mu_{1} \geq 0$ is a constant. If $u$ satisfies (1.2), then

$$
u=P_{h}[\phi]-G_{h}[\psi] \text {. }
$$

Here $P_{h}[\phi]$ and $G_{h}[\psi]$ denote the Poisson integral of $\phi$ and the Green integral of $\psi$, with respect to $\Delta_{h}$, respectively (See (2.24) and (2.25) below for the details).

The second aim of this paper is to establish the Lipschitz continuity of the mappings $u$ of the form (1.3). More precisely, we have the following.

Theorem 1.2. Let $n \geq 3$. Suppose

(1) $u \in C^{2}\left(\mathbb{B}^{n}, \mathbb{R}^{n}\right) \cap C\left(\overline{\mathbb{B}^{n}}, \mathbb{R}^{n}\right)$ is of the form (1.3);

(2) there is a constant $L \geq 0$ such that $|\phi(\xi)-\phi(\eta)| \leq L|\xi-\eta|$ for all $\xi, \eta \in \mathbb{S}^{n-1}$;

(3) there is a constant $M \geq 0$ such that $|\psi(x)| \leq M\left(1-|x|^{2}\right)$ for all $x \in \mathbb{B}^{n}$.

Then there exists a constant $N=N(n, L, M)$ such that for $x, y \in \mathbb{B}^{n}$,

$$
|u(x)-u(y)| \leq N|x-y| .
$$

Remark 1.1. In Section 6 , we give an example to show that the assumption " $n \geq 3$ " in Theorem 1.2 is necessary.

In fact, Theorem 1.2 follows from more general, albeit technical, results on Lipschitz continuity of $P_{h}[\phi]$ and $G_{h}[\psi]$, which we shall discuss next.

1.2. $\omega$-Lipschitz continuity. A continuous increasing function $\omega:[0, \infty) \rightarrow[0, \infty)$ with $\omega(0)=0$ is called a majorant if $\omega(t) / t$ is non-increasing for $t>0$. Given a subset $\Omega$ of $\mathbb{R}^{n}$, a function $f: \Omega \rightarrow \mathbb{R}^{n}$ is said to be $\omega$-Lipschitz continuous or belong to the Lipschitz space $\Lambda_{\omega}(\Omega)$ if there is a positive constant $C$ such that

$$
|f(x)-f(y)| \leq C \omega(|x-y|)
$$

for all $x, y \in \Omega$ (cf. $[9,12,13,27,28]$ ). For some $\rho_{0}>0$ and $0<\rho<\rho_{0}$, a majorant $\omega$ is called fast if

$$
\int_{0}^{\rho} \frac{\omega(t)}{t} d t \leq C \omega(\rho)
$$

Let $\Omega$ be a proper subdomain of $\mathbb{R}^{n}$. We say that a function $f: \Omega \rightarrow \mathbb{R}^{n}$ belongs to the local Lipschitz space $\operatorname{loc} \Lambda_{w}(\Omega)$ if (1.4) holds, whenever $x \in \Omega$ and $|x-y|<$ $\frac{1}{2} \delta_{\Omega}(x)$, where $C$ is a positive constant and $\delta_{\Omega}(x)$ denotes the Euclidean distance from $x$ to the boundary $\partial \Omega$ of $\Omega$.

A domain $\Omega \subset \mathbb{R}^{n}$ is said to be a $\Lambda_{w}$-extension domain if $\Lambda_{w}(\Omega)=\operatorname{loc} \Lambda_{w}(\Omega)$. In [24], Lappalainen proved that $\Omega$ is a $\Lambda_{w}$-extension domain if and only if each pair of points $x, y \in \Omega$ can be joined by a rectifiable curve $\gamma \subset \Omega$ satisfying

$$
\int_{\gamma} \frac{\omega\left(\delta_{\Omega}(\eta)\right)}{\delta_{\Omega}(\eta)} d s(\eta) \leq C \omega(|x-y|)
$$

with some fixed positive constant $C=C(\Omega, \omega)$ which means that the constant $C$ depends only on the quantities $\Omega$ and $\omega$, where $d s$ is the length measure on $\gamma$. Furthermore, we know from [24, Theorem 4.12] that $\Lambda_{w}$-extension domains exist for fast majorants $\omega$ only. Conversely, if $\omega$ is fast, then the class of $\Lambda_{\omega}$-extension domains is fairly large and contains all bounded uniform domains. 
Remark 1.2. Recall that a domain $\Omega$ is said to be uniform if there is a constant $C$ such that each pair of points $x_{1}$ and $x_{2}$ in $\Omega$ can be joined by a rectifiable curve $\gamma \subset \Omega$ satisfying

$$
\ell(\gamma) \leq C\left|x_{1}-x_{2}\right| \text { and } \min \left\{\ell\left(\gamma\left[x_{1}, x\right]\right), \ell\left(\gamma\left[x_{2}, x\right]\right)\right\} \leq C \delta_{\Omega}(x)
$$

for all $x \in \gamma$. Here $\ell(\gamma)$ denotes the length of $\gamma$ and $\left.\gamma\left[x_{i}, x\right]\right)$ is the subarc of $\gamma$ with endpoints $x_{i}$ and $x$, where $i=1,2$. It is known that $\mathbb{B}^{n}$ is a uniform domain, and hence a $\Lambda_{w}$-extension domain for a fast $\omega[13$, Section 1$]$.

The next two results establish $\omega$-Lipschitz continuity of $P_{h}[\phi]$ and $G_{h}[\psi]$ :

Theorem 1.3. Suppose $n \geq 3, \phi: \mathbb{S}^{n-1} \rightarrow \mathbb{R}^{n}$ and $|\phi(\xi)-\phi(\eta)| \leq \omega(|\xi-\eta|)$ for all $\xi, \eta \in \mathbb{S}^{n-1}$, where $\omega$ is a fast majorant. Then, for $x, y \in \mathbb{B}^{n}$,

$$
|\Phi(x)-\Phi(y)| \leq C \alpha_{0} \omega(|x-y|),
$$

where $\Phi=P_{h}[\phi]$ and $\alpha_{0}=\alpha_{0}(n)$ and $C=C\left(\mathbb{B}^{n}, \omega\right)$ is the same constant as in (1.5).

Theorem 1.4. Suppose $n \geq 3, \psi \in C\left(\mathbb{B}^{n}, \mathbb{R}^{n}\right)$ and $|\psi(x)| \leq M\left(1-|x|^{2}\right)$ for $x \in \mathbb{B}^{n}$, where $M$ is a constant. Then, for $x, y \in \mathbb{B}^{n}$,

$$
|\Psi(x)-\Psi(y)| \leq \beta_{0}|x-y|
$$

where $\Psi=G_{h}[\psi]$ and $\beta_{0}=\beta_{0}(n, M)$ is a constant.

This paper is organized as follows. In Section 2, some necessary terminology and known results are introduced, and several preliminary results are proved. In Section 3, we present the proof of Theorem 1.1. In Section 4, we show Theorem 1.3. In Section 5, we prove Theorem 1.4 and Theorem 1.2. Finally, in Section 6, we construct two examples to illustrate the necessity of the requirement $n \geq 3$ in Theorem 1.2, and the existence of hyperbolic harmonic mappings which satisfy the conditions in Theorem 1.3 but not $K$-quasiregular.

\section{Preliminaries}

In this section, we recall some necessary terminology and results.

2.1. Matrix notations. For natural number $n$, let

$$
A=\left(a_{i j}\right)_{n \times n} \in \mathbb{R}^{n \times n} .
$$

For $A \in \mathbb{R}^{n \times n}$, denote by $\|A\|$ the matrix norm $\|A\|=\sup \left\{|A x|: x \in \mathbb{R}^{n},|x|=\right.$ $1\}$, and $l(A)$ the matrix function $l(A)=\inf \left\{|A x|: x \in \mathbb{R}^{n},|x|=1\right\}$.

For a domain $\Omega \subset \mathbb{R}^{n}$, let $u=\left(u_{1}, \ldots, u_{n}\right): \Omega \rightarrow \mathbb{R}^{n}$ be a function that has all partial derivatives at $x=\left(x_{1}, \ldots, x_{n}\right)$ on $\Omega$. Then $D u$ denotes the usual Jacobian matrix

$$
D u=\left(\begin{array}{ccc}
\frac{\partial u_{1}}{\partial x_{1}} & \cdots & \frac{\partial u_{1}}{\partial x_{n}} \\
\vdots & \ddots & \vdots \\
\frac{\partial u_{n}}{\partial x_{1}} & \cdots & \frac{\partial u_{n}}{\partial x_{n}}
\end{array}\right)=\left(\nabla u_{1} \cdots \nabla u_{n}\right)^{T}
$$

where $T$ is the transpose and the gradients $\nabla u_{j}$ are understood as column vectors. If $D u$ is a nonsingular matrix, then the eigenvalues $\lambda_{j}^{2}$ of the (symmetric and positive definite) matrix $D u \times D u^{T}$ are real, and they can be ordered so that $0<\lambda_{1}^{2} \leq \lambda_{2}^{2} \leq$ 
$\ldots \leq \lambda_{n}^{2}$. Then $\left|J_{u}\right|=\prod_{k=1}^{n} \lambda_{k}, l(D u)=\lambda_{1}$ and $\|D u\|=\lambda_{n}$, where $J_{u}$ denotes the Jacobian of $u$.

2.2. Spherical coordinate transformation. Let $Q=\left(\xi_{1}, \ldots, \xi_{n}\right): K^{n-1} \rightarrow \mathbb{S}^{n-1}$ be the following spherical coordinate transformation [16]:

$$
\begin{aligned}
\xi_{1} & =\cos \theta_{1}, \\
\xi_{2} & =\sin \theta_{1} \cos \theta_{2}, \\
& \vdots \\
\xi_{n-1} & =\sin \theta_{1} \sin \theta_{2} \ldots \sin \theta_{n-2} \cos \theta_{n-1}, \\
\xi_{n} & =\sin \theta_{1} \sin \theta_{2} \ldots \sin \theta_{n-2} \sin \theta_{n-1} .
\end{aligned}
$$

Here $K^{n-1}=[0, \underbrace{\pi] \times \ldots \times[0}_{n-2}, \pi] \times[0,2 \pi]$. Note that

$$
J_{Q}\left(\theta_{1}, \ldots, \theta_{n-1}\right)=\underbrace{\sin ^{n-2} \theta_{1} \ldots \sin \theta_{n-2}}_{n-2} .
$$

For an integrable function $f$ in $\mathbb{B}^{n}$, by letting $x=\rho \xi$ with $\rho=|x|$, we have

$$
\int_{\mathbb{B}^{n}(0, r)} f(x) d \nu(x)=n \int_{0}^{r} \rho^{n-1} d \rho \int_{\mathbb{S}^{n-1}} f(\rho \xi) d \sigma(\xi),
$$

where

(see, e.g. [18, 34, 38]).

$$
d \sigma(\xi)=\frac{1}{\omega_{n-1}} J_{Q}\left(\theta_{1}, \ldots, \theta_{n-1}\right) d \theta_{1} \ldots d \theta_{n-1}
$$

2.3. $K$-quasiregular. A sense-preserving continuous mapping $u: \Omega \rightarrow G$ between two open subsets $\Omega$ and $G$ of the Euclidean space $\mathbb{R}^{n}$ will be called a $K$-quasiregular mapping $(K \geq 1)$ if

(1) $u$ is an absolutely continuous mapping in almost every segment parallel to some of the coordinate axes and there exist the partial derivatives which are locally $L^{n}$ integrable mappings on $\Omega$. We will write $u \in A C L^{n}$, and

(2) $u$ satisfies the condition $\|D u(x)\|^{n} / K \leq J_{u}(x) \leq K l(D u(x))^{n}$ at $x$ almost everywhere in $\Omega$.

In particular, $u$ is called $K$-quasiconformal if $u$ is a $K$-quasiregular homeomorphism (cf. [16, 31]).

2.4. Hypergeometric functions. Let $F$ be the hypergeometric function

$$
F(a, b ; c ; s)=\sum_{k=0}^{\infty} \frac{(a)_{k}(b)_{k}}{k !(c)_{k}} s^{k},
$$

where $a, b, c \in \mathbb{R}, c$ is neither zero nor a negative integer, $(a)_{k}$ denotes the Pochhammer symbol with $(a)_{0}=1$ and $(a)_{k}=a(a+1) \ldots(a+k-1)(k \in \mathbb{N})$. If $a$ is not a negative integer, then

$$
(a)_{k}=\Gamma(a+k) / \Gamma(a) .
$$

If $c-a-b>0$, then the series (2.5) converges absolutely for all $|s| \leq 1$ (cf. [29, Section 31]). 
Let $t>1, k \in \mathbb{R}$ and $r \in(-1,1)$. Ren and Kähler [30, Lemma 2.2] proved that

$$
\int_{-1}^{1} \frac{\left(1-s^{2}\right)^{(t-3) / 2}}{\left(1-2 r s+r^{2}\right)^{k}} d s=\frac{\Gamma\left(\frac{t-1}{2}\right) \Gamma\left(\frac{1}{2}\right)}{\Gamma\left(\frac{t}{2}\right)} F\left(k, k+1-\frac{t}{2} ; \frac{t}{2} ; r^{2}\right) .
$$

The following lemmas will be useful in the proof of Theorem 1.2.

Lemma 2.1. Let

$$
f_{n}(s)=\sum_{k=0}^{\infty} \frac{(a)_{k}\left(\frac{b-n}{2}\right)_{k}}{\left(\frac{n}{2}\right)_{k} k !} \frac{s^{k}}{k+c} .
$$

Suppose $b, n \in \mathbb{N}^{+}, a>0, c>0$ and $n-a-\frac{b}{2}>0$. Then there is a constant $\mu_{2} \geq 0$ such that for all $s \in[0,1]$ and all $n \geq b$,

$$
\left|f_{n}(s)\right| \leq \mu_{2},
$$

where $\mu_{2}=\mu_{2}(n, a, b, c)$.

Proof. Obviously, we only need to consider the case where $b$ is even since the proof of the case $b$ being odd is similar. To finish the proof, we consider the following two possibilities.

Case 2.1. $n$ is even.

Under this assumption, we easily see from $n \geq b$ that

$$
\left(\frac{b-n}{2}\right)_{k}=\left(\frac{b-n}{2}\right)\left(\frac{b-n}{2}+1\right) \ldots\left(\frac{b-n}{2}+k-1\right)=0
$$

for all $k \geq \frac{n-b+2}{2}$, and hence $f_{n}$ is a polynomial, where

$$
f_{n}(s)=\sum_{k=0}^{\frac{n-b+2}{2}} \frac{(a)_{k}\left(\frac{b-n}{2}\right)_{k}}{\left(\frac{n}{2}\right)_{k} k !} \cdot \frac{s^{k}}{k+c} .
$$

Hence, for all $s \in[0,1]$,

$$
\left|f_{n}(s)\right| \leq \mu^{\prime}, \text { where } \mu^{\prime}=\sum_{k=0}^{\frac{n-b+2}{2}} \frac{(a)_{k}\left|\left(\frac{b-n}{2}\right)_{k}\right|}{\left(\frac{n}{2}\right)_{k} k !} \frac{1}{k+c} .
$$

Case 2.2. $n$ is odd.

In this case, we separate $f_{n}$ into two parts: $f_{n}=f_{n_{1}}+f_{n_{2}}$, where

$$
f_{n_{1}}(s)=\sum_{k=0}^{\frac{n-b+1}{2}} \frac{(a)_{k}\left(\frac{b-n}{2}\right)_{k}}{\left(\frac{n}{2}\right)_{k} k !} \frac{s^{k}}{k+c}
$$

and

$$
f_{n_{2}}(s)=\sum_{k=\frac{n-b+3}{2}}^{\infty} \frac{(a)_{k}\left(\frac{b-n}{2}\right)_{k}}{\left(\frac{n}{2}\right)_{k} k !} \frac{s^{k}}{k+c}
$$


Since $f_{n_{1}}$ is continuous in $[0,1]$, obviously, for $s \in[0,1]$,

$$
\left|f_{n_{1}}(s)\right| \leq \mu^{\prime \prime}, \text { where } \mu^{\prime \prime}=\sum_{k=0}^{\frac{n-b+1}{2}} \frac{(a)_{k}\left|\left(\frac{b-n}{2}\right)_{k}\right|}{\left(\frac{n}{2}\right)_{k} k !} \frac{1}{k+c} .
$$

Next, we estimate $f_{n_{2}}$. Since $\frac{b-n}{2}+t-1>0$ for $t \geq \frac{n-b+3}{2}$ and $\frac{b-n}{2}+t-1<0$ for $t \leq \frac{n-b+1}{2}$, we obtain that

$$
f_{n_{2}}(s)=(-1)^{\frac{n-b+1}{2}} \sum_{k=\frac{n-b+3}{2}}^{\infty} \frac{(a)_{k}\left|\left(\frac{b-n}{2}\right)_{k}\right|}{\left(\frac{n}{2}\right)_{k} k !} \frac{s^{k}}{k+c} .
$$

We leave the estimate on $f_{n_{2}}$ for a moment and prove the following claim.

Claim 2.1. Let

$$
g_{a, b, n}(s)=\sum_{k=\frac{n-b+3}{2}}^{\infty} \frac{(a)_{k}\left(\frac{b-n}{2}\right)_{k}}{\left(\frac{n}{2}\right)_{k} k !} s^{k} .
$$

Then $g_{a, b, n}$ is continuous in $[0,1]$.

To prove the continuity of $g_{a, b, n}$ in $[0,1]$, it suffices to check the uniform convergence of $g_{a, b, n}$ in $[0,1]$. Since

$$
g_{a, b, n}(s)=(-1)^{\frac{n-b+1}{2}} \sum_{k=\frac{n-b+3}{2}}^{\infty} \frac{(a)_{k}\left|\left(\frac{b-n}{2}\right)_{k}\right|}{\left(\frac{n}{2}\right)_{k} k !} s^{k},
$$

obviously, we only need to demonstrate the boundedness of

$$
\sum_{k=\frac{n-b+3}{2}}^{\infty} \frac{(a)_{k}\left|\left(\frac{b-n}{2}\right)_{k}\right|}{\left(\frac{n}{2}\right)_{k} k !} .
$$

This easily follows from the following two facts:

(1) It follows from the assumption " $\frac{n}{2}-a-\frac{b-n}{2}>0$ " and [29, Section 31] that

$$
F\left(a, \frac{b-n}{2} ; \frac{n}{2} ; s\right)=\sum_{k=0}^{\infty} \frac{(a)_{k}\left(\frac{b-n}{2}\right)_{k}}{\left(\frac{n}{2}\right)_{k} k !} s^{k}
$$

is bounded in $[0,1]$.

(2) $\sum_{k=0}^{k=\frac{n-b+1}{2}} \frac{(a)_{k}\left(\frac{b-n}{2}\right)_{k}}{\left(\frac{n}{2}\right)_{k} k !} s^{k}$ is continuous in $[0,1]$.

Now, we continue the estimate on $f_{n_{2}}$. Let

$$
\left\|g_{a, b, n}\right\|_{\infty}=\max \left\{\left|g_{a, b, n}(s)\right|: s \in[0,1]\right\} .
$$

Then Claim 2.1 guarantees that $\left\|g_{a, b, n}\right\|_{\infty}$ is finite. It follows that for all $s \in[0,1]$,

$$
\left|f_{n_{2}}(s)\right| \leq\left|g_{a, b, n}(s)\right| \leq\left\|g_{a, b, n}\right\|_{\infty} .
$$

By taking

$$
\mu_{2}=\max \left\{\mu^{\prime}, \mu^{\prime \prime}+\left\|g_{a, b, n}\right\|_{\infty}\right\},
$$

the lemma follows from (2.7), (2.8) and (2.9). 
Lemma 2.2. Let

$$
I_{0}(s)=\int_{0}^{1} t^{m} F\left(a, \frac{b-n}{2} ; \frac{n}{2} ; t s\right) d t .
$$

Suppose $b, n \in \mathbb{N}^{+}, a>0$ and $m>-1$. Then for all $s \in[0,1)$,

$$
I_{0}(s)=\sum_{k=0}^{\infty} \frac{(a)_{k}\left(\frac{b-n}{2}\right)_{k}}{\left(\frac{n}{2}\right)_{k} k !} \frac{s^{k}}{k+m+1} .
$$

Proof. Obviously,

$$
\int_{0}^{1} t^{m} F\left(a, \frac{b-n}{2} ; \frac{n}{2} ; t s\right) d t=\int_{0}^{1} \sum_{k=0}^{\infty} \frac{(a)_{k}\left(\frac{b-n}{2}\right)_{k}}{\left(\frac{n}{2}\right)_{k} k !} s^{k} t^{k+m} d t,
$$

and the convergence radius of the series $\sum_{k=0}^{\infty} \frac{(a)_{k}\left(\frac{b-n}{2}\right)_{k}}{\left(\frac{n}{2}\right)_{k} k !} s^{k} t^{k+m}$ is $1 / s$ for $s \in[0,1)$. Hence we have

$$
I_{0}(s)=\sum_{k=0}^{\infty} \frac{(a)_{k}\left(\frac{b-n}{2}\right)_{k}}{\left(\frac{n}{2}\right)_{k} k !} s^{k} \int_{0}^{1} t^{k+m} d t=\sum_{k=0}^{\infty} \frac{(a)_{k}\left(\frac{b-n}{2}\right)_{k}}{\left(\frac{n}{2}\right)_{k} k !} \frac{s^{k}}{k+m+1},
$$

as required.

Lemma 2.3. Suppose $b, n \in \mathbb{N}^{+}, a>0, n-a-\frac{b}{2}>0, n \geq b$ and $m>-1$. Then for all $s \in[0,1)$,

$$
\left|I_{0}\right| \leq \mu_{2,1}
$$

where $\mu_{2,1}=\mu_{2}(n, a, b, m+1)$ and $I_{0}$ are defined in Lemmas 2.1 and 2.2, respectively.

Proof. This lemma easily follows from Lemma 2.1 and Lemma 2.2.

2.5. Möbius transformations. For any $x, y \in \mathbb{R}^{n}$, we denote the inner product $\sum_{k=1}^{n} x_{k} a_{k}$ by $\langle x, a\rangle$. Let $x=|x| x^{\prime}$ and $y=|y| y^{\prime}$. Then the symmetry lemma (see e.g. [2] or $[5,30])$ shows that

$$
|| y\left|x-y^{\prime}\right|=|| x\left|y-x^{\prime}\right|
$$

In the following, we denote $[x, y]=|| x\left|y-x^{\prime}\right|$. Obviously, $[x, y]=[y, x]$.

For any $a \in \mathbb{B}^{n}$, let

$$
\varphi_{a}(x)=\frac{|x-a|^{2} a-\left(1-|a|^{2}\right)(x-a)}{[a, x]^{2}}
$$

in $\mathbb{B}^{n}$. Then $\varphi_{a}$ is a Möbius transformation of $\mathbb{R}^{n}$ mapping $\overline{\mathbb{B}^{n}}$ onto $\overline{\mathbb{B}^{n}}$ with $\varphi_{a}(a)=$ $0, \varphi_{a}(0)=a$ and $\varphi_{a}\left(\varphi_{a}(x)\right)=x$ [36]. It follows from Equations (2.4) and (2.6), Theorem 3.4(a) and Chapter 5 in [34], together with [30, Equation (2.4)], that

$$
\begin{aligned}
{[a, x]^{2}=} & |x-a|^{2}+\left(1-|x|^{2}\right)\left(1-|a|^{2}\right) \\
= & 1+|a|^{2}|x|^{2}-2|a||x|\left\langle\frac{x}{|x|}, \frac{a}{|a|}\right\rangle, \\
& 1-|x| \leq[a, x]<2,
\end{aligned}
$$




$$
\begin{gathered}
\left|\varphi_{a}(x)\right|=\left|\varphi_{x}(a)\right|=\frac{|x-a|}{[a, x]}, \quad 1-\left|\varphi_{a}(x)\right|^{2}=\frac{\left(1-|x|^{2}\right)\left(1-|a|^{2}\right)}{[a, x]^{2}}, \\
J_{\varphi_{a}}(x)=\frac{\left(1-|a|^{2}\right)^{n}}{[a, x]^{2 n}}
\end{gathered}
$$

and

$$
\begin{aligned}
\frac{\partial}{\partial x_{k}}\left|\varphi_{a}(x)\right| & =\frac{\partial}{\partial x_{k}}\left|\varphi_{x}(a)\right| \\
& =\frac{[a, x]^{2}\left(x_{k}-a_{k}\right)-|a-x|^{2}\left(x_{k}-a_{k}\right)+|a-x|^{2}\left(1-|a|^{2}\right) x_{k}}{|a-x| \cdot[a, x]^{3}}
\end{aligned}
$$

Elementary calculations lead to

$$
\left|a-\varphi_{a}(x)\right|=\frac{\left(1-|a|^{2}\right)|x|}{[a, x]} \text { and }\left[a, \varphi_{a}(x)\right]=\frac{1-|a|^{2}}{[a, x]} .
$$

We denote by $\mathcal{M}\left(\mathbb{B}^{n}\right)$ the set of all Möbius transformations in $\mathbb{B}^{n}$. By [34, Theorem 2.1] or [36, Section 2], if $\varphi \in \mathcal{M}\left(\mathbb{B}^{n}\right)$, then there exist $a \in \mathbb{B}^{n}$ and an orthogonal transformation $A$ such that

$$
\varphi(x)=A \varphi_{a}(x) .
$$

For more information about the Möbius transformations in $\mathbb{B}^{n}$, see e.g. $[2$, Chapter 2], [6] or [37, Chapter 1].

2.6. Hyperbolic Poisson's equation. In terms of the mapping $\varphi_{a}$, the hyperbolic metric $d_{h}$ in $\mathbb{B}^{n}$ is given by

for all $a, b \in \mathbb{B}^{n}$.

$$
d_{h}(a, b)=\log \left(\frac{1+\left|\varphi_{a}(b)\right|}{1-\left|\varphi_{a}(b)\right|}\right)
$$

For all $\varphi \in \mathcal{M}\left(\mathbb{B}^{n}\right)$, by the definition of $\Delta_{h}$, we have the following Möbius invariance property [36, Section 2]:

$$
\Delta_{h}(u \circ \varphi)=\Delta_{h} u \circ \varphi
$$

Obviously,

$$
\Delta_{h} u(x)=\Delta\left(u \circ \varphi_{x}\right)(0) .
$$

In fact, if (2.17) holds for all $u \in C^{2}\left(\mathbb{B}^{n}\right)$ and $x \in \mathbb{B}^{n}$, then we can show that $\Delta_{h}$ has the representation (1.1) (cf. [34, Chapter 3] or [35]).

Let

$$
g(r, t)=\frac{1}{n} \int_{r}^{t} \frac{\left(1-s^{2}\right)^{n-2}}{s^{n-1}} d s \quad \text { and } \quad g(r)=g(r, 1),
$$

where $0 \leq r<t<1$. It is well known that the Green's function $G_{h}(x, y)$ w.r.t. $\Delta_{h}$ is given by

$$
G_{h}(x, y)=g\left(\left|\varphi_{x}(y)\right|\right)=\frac{1}{n} \int_{\left|\varphi_{x}(y)\right|}^{1} \frac{\left(1-s^{2}\right)^{n-2}}{s^{n-1}} d s
$$

for all $x \neq y \in \mathbb{B}^{n}$. 
We remark that in the complex plane $\mathbb{C}$, every Möbius transformation $\varphi$ mapping the unit disc $\mathbb{D}$ onto itself can be written as $\varphi(z)=e^{i \theta} \varphi_{w}(z)$, where $\varphi_{w}(z)=\frac{w-z}{1-\bar{w} z}$ for some $w$ in $\mathbb{D}$. Hence when $n=2$, by (2.19), we get [22]

$$
G_{h}(w, z)=g\left(\left|\varphi_{w}(z)\right|\right)=\frac{1}{2} \log \frac{|1-\bar{w} z|}{|w-z|}=\pi \cdot G(w, z),
$$

where $G$ is the usual Green function w.r.t. $\Delta$.

For function $g$ in (2.18), we define

$$
q(t)=\frac{t^{n-2}}{\left(1-t^{2}\right)^{n-1}} g(t)
$$

in $(0,1)$. Since elementary calculations lead to

$$
\lim _{t \rightarrow 0^{+}} q(t)=\frac{1}{n(n-2)} \text { and } \lim _{t \rightarrow 1^{-}} q(t)=\frac{1}{2 n(n-1)},
$$

we define

Then we have

$$
q(0)=\frac{1}{n(n-2)} \text { and } q(1)=\frac{1}{2 n(n-1)} .
$$

Lemma 2.4. For $n \geq 3, \frac{1}{2 n(n-1)} \leq q(t) \leq \frac{1}{n(n-2)}$ in $[0,1]$.

Proof. We start with the following claim.

Claim 2.2. For $n \geq 3, \frac{1}{2 n(n-1)}<q(t)<\frac{1}{n(n-2)}$ in $(0,1)$.

For $t \in(0,1]$, let

$$
q_{1}(t)=g(t)-\frac{1}{n(n-2)} \cdot \frac{\left(1-t^{2}\right)^{n-1}}{t^{n-2}}
$$

and

$$
q_{2}(t)=g(t)-\frac{1}{2 n(n-1)} \cdot \frac{\left(1-t^{2}\right)^{n-1}}{t^{n-2}} .
$$

Then $q_{1}(t)$ is increasing and $q_{2}(t)$ is decreasing, respectively, in $(0,1)$. Since $q_{1}(1)=$ $q_{2}(1)=0$, we see that

$$
\frac{1}{2 n(n-1)} \cdot \frac{\left(1-t^{2}\right)^{n-1}}{t^{n-2}} \leq g(t) \leq \frac{1}{n(n-2)} \cdot \frac{\left(1-t^{2}\right)^{n-1}}{t^{n-2}}
$$

in $(0,1]$, which implies that the claim holds.

Now, the lemma easily follows from Claim 2.1 and (2.21).

The Poisson-Szegő kernel $P_{h}$ for $\Delta_{h}$ is given by

$$
P_{h}(x, t)=\left(\frac{1-|x|^{2}}{|t-x|^{2}}\right)^{n-1},
$$

which satisfies (cf. [34, Lemma 5.20] or [35])

$$
\int_{\mathbb{S}^{n-1}} P_{h}(x, t) d \sigma(t)=1
$$


and for each $k \in\{1,2, \ldots, n\}$,

$$
\begin{aligned}
& \frac{\partial}{\partial x_{k}} P_{h}(x, t)=\frac{\partial}{\partial x_{k}}\left(\frac{1-|x|^{2}}{|t-x|^{2}}\right)^{n-1} \\
= & -2(n-1) \frac{x_{k}|t-x|^{2}+\left(1-|x|^{2}\right)\left(x_{k}-t_{k}\right)}{|t-x|^{4}} \cdot\left(\frac{1-|x|^{2}}{|t-x|^{2}}\right)^{n-2},
\end{aligned}
$$

where $(x, t) \in \mathbb{B}^{n} \times \mathbb{S}^{n-1}$.

If $\phi \in L^{1}\left(\mathbb{S}^{n-1}, \mathbb{R}^{n}\right)(n \geq 2)$, we define the Poisson-Szegő integral or invariant Poisson integral of $\phi$ (cf. $[1,14,35]$ or [34, Definition 5.21]) by

$$
P_{h}[\phi](x)=\int_{\mathbb{S}^{n-1}} P_{h}(x, \xi) \phi(\xi) d \sigma(\xi) .
$$

If $\psi$ satisfies the following conditions:

(1) For $n \geq 3, \psi \in C\left(\mathbb{B}^{n}, \mathbb{R}^{n}\right)$ and $\int_{\mathbb{B}^{n}}\left(1-|x|^{2}\right)^{n-1}|\psi(x)| d \tau(x)<\infty$,

(2) For $n=2, \psi(z)=\left(1-|z|^{2}\right)^{2} \psi_{0}(z)$, where $\psi_{0} \in C(\mathbb{D}, \mathbb{C})$,

then we define a function $G_{h}[\psi]$ by

$$
\begin{aligned}
G_{h}[\psi](x) & =\int_{\mathbb{B}^{n}} G_{h}(x, y) \psi(y) d \tau(y) \\
& =\frac{1}{n} \int_{\mathbb{B}^{n}}\left[\psi(y) \int_{\left|\varphi_{x}(y)\right|}^{1} \frac{\left(1-s^{2}\right)^{n-2}}{s^{n-1}} d s\right] d \tau(y) .
\end{aligned}
$$

This function is called the invariant Green integral of $\psi$.

Remark 2.1. If $n=2$ and $\Delta u(z) \in C(\mathbb{D}, \mathbb{C})$, then it follows from (2.20), together with the facts $\Delta_{h} u(z)=\left(1-|z|^{2}\right)^{2} \Delta u(z)$ and $d \tau(z)=\frac{1}{\pi}\left(1-|z|^{2}\right)^{-2} d A(z)$, that

$$
G_{h}\left[\Delta_{h} u\right](z)=\frac{1}{2 \pi} \int_{\mathbb{D}} \log \frac{|1-\bar{w} z|}{|w-z|} \Delta_{h} u(z) \frac{d A(z)}{\left(1-|z|^{2}\right)^{2}}=G[\Delta u](z),
$$

where $d A\left(r e^{i \theta}\right)=r d r d \theta$.

Furthermore, (2.22) implies that $P_{h}=P$ provided that $n=2$. Let

$$
\psi(z)=\left(1-|z|^{2}\right)^{2} \psi_{0}(z),
$$

where $\psi_{0} \in C(\mathbb{D}, \mathbb{C})$. If $u$ satisfies $\Delta u=\psi_{0}$ in $\mathbb{D}$ and $\left.u\right|_{\mathbb{S}^{1}}=\phi \in L^{1}\left(\mathbb{S}^{1}, \mathbb{C}\right)$, then it follows from $[22],(2.24)$ and (2.26) that

$$
u=P[\phi]-G\left[\psi_{0}\right]=P_{h}[\phi]-G_{h}[\psi] .
$$

We use $C_{c}^{2}\left(\mathbb{B}^{n}\right)$ to denote the set of all twice continuous differentiable functions with compact support in $\mathbb{B}^{n}$. Let us recall the following two results.

Theorem A. ([34, Corollary 4.4] or [35]) If $u \in C_{c}^{2}\left(\mathbb{B}^{n}, \mathbb{R}^{n}\right)$, then for all $x \in \mathbb{B}^{n}$,

$$
u=-G_{h}\left[\Delta_{h} u\right] .
$$


Theorem B. ([34, Theorem 5.22] or [35]) Let $\phi \in C\left(\mathbb{S}^{n-1}, \mathbb{R}^{n}\right)$, and let $F$ be defined as follows:

$$
F(x)=\left\{\begin{array}{cl}
P_{h}[\phi](x), & x \in \mathbb{B}^{n}, \\
\phi(x), & x \in \mathbb{S}^{n-1} .
\end{array}\right.
$$

Then (1) $F$ is hyperbolically harmonic in $\mathbb{B}^{n}$ and continuous in $\overline{\mathbb{B}^{n}}$;

(2) $\|F\|_{\infty}=\|\phi\|_{\infty}$, where $\|F\|_{\infty}=\sup \left\{|F(x)|: x \in \overline{\mathbb{B}^{n}}\right\}$ and $\|\phi\|_{\infty}=\sup \{|\phi(\xi)|:$ $\left.\xi \in \mathbb{S}^{n-1}\right\}$.

Conversely, if $H$ is hyperbolically harmonic in $\mathbb{B}^{n}$ and continuous in $\overline{\mathbb{B}^{n}}$, then

$$
H=P_{h}[H] \text {. }
$$

\section{Representation of SOlutions to $\Delta_{h} u=\psi$}

The main purpose of this section is to prove Theorem 1.1. In this section, we always assume that $n \geq 3$. Before the proof, we recall the following results.

Theorem C. ([36, Lemma 3.2]) If $u \in C^{2}\left(\mathbb{B}^{n}\right)$, then

$$
u(0)=\int_{\mathbb{S}^{n-1}} u(r \xi) d \sigma(\xi)-\int_{\mathbb{B}^{n}(0, r)} g(|x|, r) \Delta_{h} u(x) d \tau(x),
$$

where $0<r<1$ and $g(t, r)$ is defined in (2.18).

Theorem D. ([36, Corollary 4.1]) For any $y \in \mathbb{B}^{n}$,

$$
\int_{\mathbb{B}^{n}} G_{h}(x, y) d \nu(x)=\frac{1}{2 n(n-1)}\left(1-|y|^{2}\right)^{n-1} .
$$

The next two theorems are about the Möbius invariance of $P_{h}[f]$ and $d \tau$.

Theorem E. ([34, Theorem 5.23] or [35]) If $f \in L^{1}\left(\mathbb{S}^{n-1}\right)$, then

$$
P_{h}[f \circ \varphi]=P_{h}[f] \circ \varphi
$$

for all $\varphi \in \mathcal{M}\left(\mathbb{B}^{n}\right)$.

Theorem F. ([34, Theorem 3.4(b)] or [35]) If $f \in L^{1}\left(\mathbb{B}^{n}, \tau\right)$ and $\varphi \in \mathcal{M}\left(\mathbb{B}^{n}\right)$, then

$$
\int_{\mathbb{B}^{n}} f(x) d \tau(x)=\int_{\mathbb{B}^{n}} f \circ \varphi(y) d \tau(y) .
$$

Lemma 3.1. Let $\psi \in C\left(\mathbb{B}^{n}, \mathbb{R}^{n}\right)$. Suppose there is a constant $\mu_{1}$ such that

$$
\int_{\mathbb{B}^{n}}\left(1-|x|^{2}\right)^{n-1}|\psi(x)| d \tau(x) \leq \mu_{1} .
$$

Then

$$
\int_{\mathbb{B}^{n}} g(|x|)|\psi(x)| d \tau(x) \leq \mu_{3}
$$


where $g(r)$ is defined in (2.18), $\mu_{3}=\mu_{3}\left(n, \mu_{1},\|\psi\|_{\frac{1}{2}, \infty}\right)$ and $\|\psi\|_{\frac{1}{2}, \infty}=\sup \{|\psi(x)|$ : $\left.x \in \mathbb{B}^{n}\left(0, \frac{1}{2}\right)\right\}$.

Proof. By letting $y=0$ in (2.19) and Theorem D, we get

$$
\int_{\mathbb{B}^{n}\left(0, \frac{1}{2}\right)} g(|x|) d \tau(x)=\int_{\mathbb{B}^{n}\left(0, \frac{1}{2}\right)} G_{h}(x, 0) \frac{d \nu(x)}{\left(1-|x|^{2}\right)^{n}} \leq \frac{1}{2 n(n-1)\left(1-\frac{1}{4}\right)^{n}},
$$

and thus the assumption " $\psi \in C\left(\mathbb{B}^{n}, \mathbb{R}^{n}\right)$ " gives that

$$
\int_{\mathbb{B}^{n}\left(0, \frac{1}{2}\right)} g(|x|)|\psi(x)| d \tau(x) \leq \frac{\|\psi\|_{\frac{1}{2}, \infty}}{2 n(n-1)\left(1-\frac{1}{4}\right)^{n}} .
$$

Obviously, for $x \in \mathbb{B}^{n} \backslash \mathbb{B}^{n}\left(0, \frac{1}{2}\right)$,

$$
g(|x|)=\frac{1}{2 n} \int_{|x|}^{1} \frac{\left(1-s^{2}\right)^{n-2}}{s^{n}} d s^{2} \leq \frac{2^{n-1}}{n} \int_{|x|}^{1}\left(1-s^{2}\right)^{n-2} d s^{2}=\frac{2^{n-1}}{n(n-1)}\left(1-|x|^{2}\right)^{n-1},
$$

it follows that

Since

$$
\int_{\mathbb{B}^{n} \backslash \mathbb{B}^{n}\left(0, \frac{1}{2}\right)} g(|x|)|\psi(x)| d \tau(x) \leq \frac{2^{n-1}}{n(n-1)} \mu_{1} .
$$

$$
\int_{\mathbb{B}^{n}} g(|x|)|\psi(x)| d \tau(x)=\int_{\mathbb{B}^{n}\left(0, \frac{1}{2}\right)} g(|x|)|\psi(x)| d \tau(x)+\int_{\mathbb{B}^{n} \backslash \mathbb{B}^{n}\left(0, \frac{1}{2}\right)} g(|x|)|\psi(x)| d \tau(x),
$$

by letting

$$
\mu_{3}=\frac{\|\psi\|_{\frac{1}{2}, \infty}}{2 n(n-1)\left(1-\frac{1}{4}\right)^{n}}+\frac{2^{n-1}}{n(n-1)} \mu_{1},
$$

we see that the lemma holds.

Lemma 3.2. Suppose that $u \in C^{2}\left(\mathbb{B}^{n}, \mathbb{R}^{n}\right) \cap C\left(\overline{\mathbb{B}^{n}}, \mathbb{R}^{n}\right)$ and satisfies (1.2). If

$$
\int_{\mathbb{B}^{n}}\left(1-|x|^{2}\right)^{n-1}|\psi(x)| d \tau(x) \leq \mu_{1}
$$

then

$$
u(0)=P_{h}[\phi](0)-\int_{\mathbb{B}^{n}} G_{h}(0, x) \psi(x) d \tau(x) .
$$

Proof. It follows from the assumption " $\int_{\mathbb{B}^{n}}\left(1-|x|^{2}\right)^{n-1}|\psi(x)| d \tau(x) \leq \mu_{1}$ " and Lemma 3.1 that

$$
\int_{\mathbb{B}^{n}} g(|x|)|\psi(x)| d \tau(x) \leq \mu_{3}
$$

Since

$$
\int_{\mathbb{B}^{n}(0, r)} g(|x|, r)|\psi(x)| d \tau(x) \leq \int_{\mathbb{B}^{n}} g(|x|)|\psi(x)| d \tau(x),
$$

by Lebesgue's Dominated Convergence Theorem, we have that

$$
\lim _{r \rightarrow 1^{-}} \int_{\mathbb{B}^{n}(0, r)} g(|x|, r) \psi(x) d \tau(x)=\int_{\mathbb{B}^{n}} g(|x|) \psi(x) d \tau(x) .
$$


Furthermore, the assumption " $u \in C\left(\overline{\mathbb{B}^{n}}, \mathbb{R}^{n}\right)$ " gives that

$$
\lim _{r \rightarrow 1^{-}} \int_{\mathbb{S}^{n-1}} u(r \xi) d \sigma(\xi)=\int_{\mathbb{S}^{n-1}} u(\xi) d \sigma(\xi) .
$$

Then Theorem C, (3.1) and (3.2) imply that

$$
\begin{aligned}
u(0) & =\int_{\mathbb{S}^{n-1}} u(\xi) d \sigma(\xi)-\int_{\mathbb{B}^{n}} g(|x|) \Delta_{h} u(x) d \tau(x) \\
& =\int_{\mathbb{S}^{n-1}} P_{h}(0, \xi) \phi(\xi) d \sigma(\xi)-\int_{\mathbb{B}^{n}} G_{h}(0, x) \psi(x) d \tau(x),
\end{aligned}
$$

as required.

Proof of Theorem 1.1. We prove this theorem by two steps. In the first step, we check that for any fixed $\zeta \in \overline{\mathbb{B}^{n}}\left(0, r_{0}\right), u \circ \varphi_{\zeta}$ satisfies the requirements in Lemma 3.2 , where $0 \leq r_{0}<1$. In the second step, by applying Lemma 3.2 to $u \circ \varphi_{\zeta}$, we finish the proof.

Claim 3.1. For any fixed $\zeta \in \overline{\mathbb{B}^{n}}\left(0, r_{0}\right), u \circ \varphi_{\zeta}$ satisfies the requirements in Lemma 3.2.

Obviously, for any fixed $\zeta \in \overline{\mathbb{B}^{n}}\left(0, r_{0}\right), u \circ \varphi_{\zeta} \in C^{2}\left(\mathbb{B}^{n}, \mathbb{R}^{n}\right) \cap C\left(\overline{\mathbb{B}^{n}}, \mathbb{R}^{n}\right)$. The Möbius invariance property (2.16) and the assumption " $u \in C\left(\overline{\mathbb{B}^{n}}, \mathbb{R}^{n}\right)$ " imply that

$$
\Delta_{h}\left(u \circ \varphi_{\zeta}\right)(y)=\Delta_{h} u\left(\varphi_{\zeta}(y)\right)=\psi\left(\varphi_{\zeta}(y)\right)=\psi \circ \varphi_{\zeta}(y)
$$

in $\mathbb{B}^{n}$ and

$$
\left.\left(u \circ \varphi_{\zeta}\right)\right|_{\mathbb{S}^{n-1}}=\phi \circ \varphi_{\zeta} .
$$

So, to prove the claim, it suffices to show the following: There exists a constant $\mu_{4}$ such that

$$
\int_{\mathbb{B}^{n}}\left(1-|y|^{2}\right)^{n-1}\left|\psi\left(\varphi_{\zeta}(y)\right)\right| d \tau(y) \leq \mu_{4},
$$

where $\mu_{4}=\mu_{4}\left(\mu_{1}, n, r_{0}\right)$.

Let $w=\varphi_{\zeta}(y)$. Then we have that $y=\varphi_{\zeta}(w)$, so Theorem F, (2.11) and (2.12), together with the assumption " $\int_{\mathbb{B}^{n}}\left(1-|x|^{2}\right)^{n-1}|\psi(x)| d \tau(x) \leq \mu_{1}$ ", yield

$$
\begin{aligned}
& \int_{\mathbb{B}^{n}}\left(1-|y|^{2}\right)^{n-1}\left|\psi\left(\varphi_{\zeta}(y)\right)\right| d \tau(y) \\
= & \left.\int_{\mathbb{B}^{n}}\left(1-\left|\varphi_{\zeta}(w)\right|^{2}\right)^{n-1}|\psi(w)| d \tau(w) \quad \text { (letting } w=\varphi_{\zeta}(y)\right) \\
\leq & \left.2^{n-1} \int_{\mathbb{B}^{n}} \frac{\left(1-|w|^{2}\right)^{n-1}|\psi(w)|}{(1-|\zeta|)^{n-1}} d \tau(w) \quad \text { (by (2.11) and }(2.12)\right) \\
\leq & \frac{2^{n-1} \mu_{1}}{\left(1-r_{0}\right)^{n-1}} .
\end{aligned}
$$

Obviously, letting $\mu_{4}=2^{n-1} \mu_{1}\left(1-r_{0}\right)^{1-n}$ yields (3.3).

Claim 3.2. $u=P_{h}[\phi]-G_{h}[\psi]$. 
By replacing $u$ with $u \circ \varphi_{\zeta}$ and by using (2.16) and Theorem E, we see from Lemma 3.2 that

$$
\begin{aligned}
u(\zeta) & =u \circ \varphi_{\zeta}(0)=P_{h}\left[\phi \circ \varphi_{\zeta}\right](0)-\int_{\mathbb{B}^{n}} G_{h}(0, y) \Delta_{h}\left(u \circ \varphi_{\zeta}\right)(y) d \tau(y) \\
& =P_{h}[\phi]\left(\varphi_{\zeta}(0)\right)-\int_{\mathbb{B}^{n}} G_{h}(0, y) \Delta_{h} u\left(\varphi_{\zeta}(y)\right) d \tau(y) .
\end{aligned}
$$

Let $w=\varphi_{\zeta}(y)$. It follows from

$$
\varphi_{\zeta}(0)=\zeta, \quad G_{h}\left(0, \varphi_{\zeta}(w)\right)=g\left(\left|\varphi_{\zeta}(w)\right|\right)=G_{h}(\zeta, w)
$$

and Theorem $\mathrm{F}$ that

$$
u(\zeta)=P_{h}[\phi](\zeta)-\int_{\mathbb{B}^{n}} G_{h}(\zeta, w) \Delta_{h} u(w) d \tau(w)=P_{h}[\phi](\zeta)-G_{h}[\psi](\zeta) .
$$

By the arbitrariness of $r_{0}$ in $[0,1)$, we see that the proof of the theorem is complete.

\section{Lipschitz CONTINUity of $\Phi=P_{h}[\phi]$}

The aim of this section is to prove the $\omega$-Lipschitz continuity of $\Phi=P_{h}[\phi]$ (Theorem 1.3).

Before the proof of Theorem 1.3, we need an estimate on $\|D \Phi(x)\|$ in terms of $\omega(1-|x|)$ which is formulated in Lemma 4.4. The proof of Lemma 4.4 needs some preparation which consists of three lemmas. The first lemma is as follows.

Lemma 4.1. Suppose $\phi \in C\left(\mathbb{S}^{n-1}, \mathbb{R}^{n}\right)$. Then for each $k \in\{1,2, \ldots, n\}$,

(1) $\frac{\partial}{\partial x_{k}} \Phi(x)$ is continuous in $\mathbb{B}^{n}$;

(2) $\frac{\partial}{\partial x_{k}} \Phi(x)=\int_{\mathbb{S}^{n-1}} \frac{\partial}{\partial x_{k}} P_{h}(x, \xi) \phi(\xi) d \sigma(\xi)$ for $x \in \mathbb{B}^{n}$.

Proof. In order to prove this lemma, we only need to discuss the case $k=1$ since other cases can be discussed in a similar way. For this, we assume that $x \in \overline{\mathbb{B}^{n}}\left(0, r_{0}\right)$ and $x+\Delta x_{1} \in \overline{\mathbb{B}^{n}}\left(0, r_{0}\right)$, where $x=\left(x_{1}, \ldots, x_{n}\right), x+\Delta x_{1}=\left(x_{1}+\Delta x_{1}, \ldots, x_{n}\right)$ and $0<r_{0}<1$. Then

$$
\frac{\Phi\left(x+\Delta x_{1}\right)-\Phi(x)}{\Delta x_{1}}=\int_{\mathbb{S}^{n-1}} \frac{P_{h}\left(x+\Delta x_{1}, \xi\right)-P_{h}(x, \xi)}{\Delta x_{1}} \phi(\xi) d \sigma(\xi) .
$$

Obviously, $\frac{\partial}{\partial x_{1}} P_{h}(x, \xi) \phi(\xi)$ is continuous in $\overline{\mathbb{B}^{n}}\left(0, r_{0}\right) \times \mathbb{S}^{n-1}$, and so

$$
\int_{\mathbb{S}^{n-1}} \frac{\partial}{\partial x_{1}} P_{h}(x, \xi) \phi(\xi) d \sigma(\xi)
$$

is continuous on $\overline{\mathbb{B}^{n}}\left(0, r_{0}\right)$. By applying the Lagrange mean-value theorem to $P_{h}(x, \xi)$ w.r.t. $x_{1}$, we see that there exists $t_{1} \in(0,1)$ such that

$$
\begin{aligned}
\frac{\partial \Phi(x)}{\partial x_{1}} & =\lim _{\Delta x_{1} \rightarrow 0} \frac{\Phi\left(x+\Delta x_{1}\right)-\Phi(x)}{\Delta x_{1}}=\lim _{\Delta x_{1} \rightarrow 0} \int_{\mathbb{S}^{n-1}} \frac{\partial}{\partial x_{1}} P_{h}\left(x+t_{1} \Delta x_{1}, \xi\right) \phi(\xi) d \sigma(\xi) \\
& =\int_{\mathbb{S}^{n-1}} \frac{\partial}{\partial x_{1}} P_{h}(x, \xi) \phi(\xi) d \sigma(\xi),
\end{aligned}
$$


as required.

Let $\xi_{0}=e_{1} \in \mathbb{S}^{n-1}$ denote the first unit coordinate vector $(1,0, \ldots, 0)$. Then we have the following estimate.

Lemma 4.2. Suppose $q \geq 0, p-q-n>0$ and $n \geq 3$. Then

$$
\begin{aligned}
& \int_{\mathbb{S}^{n-1}} \frac{\left|\xi-\xi_{0}\right|^{q} \omega\left(\left|\xi-\xi_{0}\right|\right)}{\left|\xi-r \xi_{0}\right|^{p}} d \sigma(\xi) \\
\leq & \alpha_{1} \frac{\omega(1-r)}{(1-r)^{p+1-q-n}}\left(\frac{1}{q+n-1}+\frac{2^{p}}{p-q-n}\right),
\end{aligned}
$$

where $\omega$ is a majorant, $0 \leq r<1$ and $\alpha_{1}=\omega_{n-2} / \omega_{n-1}=\frac{1}{\sqrt{\pi}} \frac{\Gamma\left(\frac{n}{2}\right)}{\Gamma\left(\frac{n-1}{2}\right)}$.

Proof. We shall prove this lemma by using a similar argument as in [3] and [4]. In order to estimate the integral in (4.1), we split $\mathbb{S}^{n-1}$ into the following two subsets:

$$
E=\left\{\xi \in \mathbb{S}^{n-1}:\left|\xi-\xi_{0}\right| \leq 1-r\right\} \text { and } F=\left\{\xi \in \mathbb{S}^{n-1}:\left|\xi-\xi_{0}\right|>1-r\right\} .
$$

Then (4.1) easily follows from the following two claims.

Claim 4.1. $\int_{E} \frac{\left|\xi-\xi_{0}\right|^{q} \omega\left(\left|\xi-\xi_{0}\right|\right)}{\left|\xi-r \xi_{0}\right|^{p}} d \sigma(\xi) \leq \alpha_{1} \frac{\omega(1-r)}{q+n-1}(1-r)^{q+n-1-p}$.

Since $\left|\xi-r \xi_{0}\right| \geq 1-\left|r \xi_{0}\right|=1-r$ for all $\xi \in \mathbb{S}^{n-1}$, we have

$$
\begin{aligned}
\int_{E} \frac{\left|\xi-\xi_{0}\right|^{q} \omega\left(\left|\xi-\xi_{0}\right|\right)}{\left|\xi-r \xi_{0}\right|^{p}} d \sigma(\xi) & \leq \int_{E} \frac{\left|\xi-\xi_{0}\right|^{q} \omega\left(\left|\xi-\xi_{0}\right|\right)}{(1-r)^{p}} d \sigma(\xi) \\
& =\frac{(1-r)^{-p}}{\omega_{n-1}} \int_{E}\left|\xi-\xi_{0}\right|^{q} \omega\left(\left|\xi-\xi_{0}\right|\right) d S(\xi),
\end{aligned}
$$

where $S$ denotes the $(n-1)$-dimensional Lebesgue measure on $\mathbb{S}^{n-1}$. Let $\xi=$ $\left(\xi_{1}, \ldots, \xi_{n}\right) \in E$ has the expression (2.2). Then, $\theta_{1} \in\left[0, \varphi_{r}\right] \subset\left[0, \frac{\pi}{2}\right], \theta_{2}, \ldots, \theta_{n-2} \in$ $[0, \pi]$ and $\theta_{n-1} \in[0,2 \pi]$, where $\varphi_{r}=2 \arcsin \frac{1-r}{2}$. It follows from $(2.3)$ that

$$
\begin{aligned}
\int_{E}\left|\xi-\xi_{0}\right|^{q} \omega\left(\left|\xi-\xi_{0}\right|\right) d S(\xi)= & \int_{0}^{\varphi_{r}}\left(2-2 \cos \theta_{1}\right)^{\frac{q}{2}} \omega\left(\left(2-2 \cos \theta_{1}\right)^{\frac{1}{2}}\right) \sin ^{n-2} \theta_{1} d \theta_{1} \\
& \cdot \int_{0}^{\pi} \sin ^{n-3} \theta_{2} d \theta_{2} \cdots \int_{0}^{\pi} \sin \theta_{n-2} d \theta_{n-2} \int_{0}^{2 \pi} d \theta_{n-1} \\
= & \omega_{n-2} \int_{0}^{\varphi_{r}}\left(2-2 \cos \theta_{1}\right)^{\frac{q}{2}} \omega\left(\left(2-2 \cos \theta_{1}\right)^{\frac{1}{2}}\right) \sin ^{n-2} \theta_{1} d \theta_{1} .
\end{aligned}
$$

Let $\rho=\sqrt{2-2 \cos \theta_{1}} \in[0,1-r]$. Then $d \theta_{1}=\frac{\rho}{\sin \theta_{1}} d \rho$, from which we deduce that

$$
\begin{aligned}
& \int_{E}\left|\xi-\xi_{0}\right|^{q} \omega\left(\left|\xi-\xi_{0}\right|\right) d S(\xi) \\
\leq & \omega_{n-2} \int_{0}^{1-r} \rho^{q+n-2} \omega(\rho) d \rho \leq \frac{\omega_{n-2} \omega(1-r)}{q+n-1}(1-r)^{q+n-1},
\end{aligned}
$$


where in the first inequality, the relation $\sin ^{2} \theta_{1}=\rho^{2}\left(1-\frac{\rho^{2}}{4}\right) \leq \rho^{2}$ is applied. It follows from $\alpha_{1}=\omega_{n-2} / \omega_{n-1},(4.2)$ and (4.3) that

$$
\int_{E} \frac{\left|\xi-\xi_{0}\right|^{q} \omega\left(\left|\xi-\xi_{0}\right|\right)}{\left|\xi-r \xi_{0}\right|^{p}} d \sigma(\xi) \leq \alpha_{1} \frac{\omega(1-r)}{q+n-1}(1-r)^{q+n-1-p},
$$

which is what we need.

Claim 4.2. $\int_{F} \frac{\left|\xi-\xi_{0}\right|^{q}}{\left|r \xi_{0}-\xi\right|^{p}} \omega\left(\left|\xi-\xi_{0}\right|\right) d \sigma(\xi) \leq 2^{p} \alpha_{1} \frac{\omega(1-r)}{p-q-n}(1-r)^{q+n-1-p}$.

Since for all $\xi \in F$,

$$
\left|\xi-\xi_{0}\right| \leq\left|\xi-r \xi_{0}\right|+\left|r \xi_{0}-\xi_{0}\right|=\left|\xi-r \xi_{0}\right|+1-r \text { and }\left|\xi-r \xi_{0}\right| \geq 1-r,
$$

we easily see that

$$
\left|\xi-\xi_{0}\right| \leq 2\left|\xi-r \xi_{0}\right|
$$

and so

$$
\int_{F} \frac{\left|\xi-\xi_{0}\right|^{q} \omega\left(\left|\xi-\xi_{0}\right|\right)}{\left|\xi-r \xi_{0}\right|^{p}} d \sigma(\xi) \leq 2^{p} \int_{F}\left|\xi-\xi_{0}\right|^{q-p} \omega\left(\left|\xi-\xi_{0}\right|\right) d \sigma(\xi) .
$$

Then the similar reasoning as in the proof of (4.3) leads to

$$
\begin{aligned}
\int_{F} \frac{\left|\xi-\xi_{0}\right|^{q}}{\left|r \xi_{0}-\xi\right|^{p}} \omega\left(\left|\xi-\xi_{0}\right|\right) d \sigma(\xi) & \leq 2^{p} \alpha_{1} \int_{1-r}^{2} \rho^{q-p} \omega(\rho) \rho^{n-2} d \rho \\
& \leq \frac{2^{p} \alpha_{1} \omega(1-r)}{p-q-n}(1-r)^{q+n-1-p}
\end{aligned}
$$

where, in the last inequality, the assumption that $\frac{\omega(t)}{t}$ is non-increasing, is exploited. Hence Claim 4.2 is true.

Based on Lemmas 4.1 and 4.2, we have the following estimate on $\left|\frac{\partial \Phi}{\partial x_{k}}(x)\right|$.

Lemma 4.3. Suppose $\phi$ and $\omega$ satisfy the conditions in Theorem 1.3. Then there is a constant $\alpha_{2}$ such that for all $x \in\left[0, e_{1}\right)$ and $k \in\{1,2, \ldots, n\}$,

$$
\left|\frac{\partial \Phi}{\partial x_{k}}(x)\right| \leq \alpha_{2} \frac{\omega(1-r)}{1-r}
$$

where $\alpha_{2}=\alpha_{2}(n),\left[0, e_{1}\right)=\left\{x \in \mathbb{B}^{n}: x=r e_{1}, 0 \leq r<1\right\}$ and $n \geq 3$.

Proof. For any $x_{0} \in\left[0, e_{1}\right)$, obviously, there is an $r \in[0,1)$ such that $x_{0}=r \xi_{0}$, where $\xi_{0}=e_{1}$. We prove the claim by considering two cases.

Case 4.1. $2 \leq k \leq n$.

Since (2.23) implies

$$
\frac{\partial}{\partial x_{k}} P_{h}\left(x_{0}, \xi\right)=\frac{2(n-1)\left(1-\left|x_{0}\right|^{2}\right)^{n-1} \xi_{k}}{\left|\xi-x_{0}\right|^{2 n}},
$$

we infer from

$$
\phi\left(\xi_{0}\right)=\int_{\mathbb{S}^{n-1}} P_{h}(x, \xi) \phi\left(\xi_{0}\right) d \sigma(\xi),
$$


together with Lemma 4.1, that

$$
\begin{aligned}
\left|\frac{\partial \Phi}{\partial x_{k}}\left(x_{0}\right)\right| & =\left|\int_{\mathbb{S}^{n-1}} \frac{\partial}{\partial x_{k}} P_{h}\left(x_{0}, \xi\right) \phi(\xi) d \sigma(\xi)\right| \\
& =\left|\int_{\mathbb{S}^{n-1}} \frac{2(n-1)\left(1-\left|x_{0}\right|^{2}\right)^{n-1} \xi_{k}}{\left|\xi-x_{0}\right|^{2 n}}\left(\phi(\xi)-\phi\left(\xi_{0}\right)\right) d \sigma(\xi)\right| \\
& \leq 2(n-1)\left(1-\left|x_{0}\right|^{2}\right)^{n-1} \int_{\mathbb{S}^{n-1}} \frac{\left|\xi_{k}\right| \cdot\left|\phi(\xi)-\phi\left(\xi_{0}\right)\right|}{\left|\xi-x_{0}\right|^{2 n}} d \sigma(\xi) .
\end{aligned}
$$

By using the fact $\left|\xi_{k}\right| \leq\left|\xi-\xi_{0}\right|$ for $2 \leq k \leq n$ and the assumption " $\left|\phi(\xi)-\phi\left(\xi_{0}\right)\right| \leq$ $\omega\left(\left|\xi-\xi_{0}\right|\right) "$, we get

$$
\left|\frac{\partial \Phi}{\partial x_{k}}\left(x_{0}\right)\right| \leq 2(n-1)\left(1-\left|x_{0}\right|^{2}\right)^{n-1} \int_{\mathbb{S}^{n-1}} \frac{\left|\xi-\xi_{0}\right| \omega\left(\left|\xi-\xi_{0}\right|\right)}{\left|\xi-x_{0}\right|^{2 n}} d \sigma(\xi) .
$$

Then Lemma 4.2 leads to

$$
\left|\frac{\partial \Phi}{\partial x_{k}}\left(r e_{1}\right)\right| \leq \alpha_{3} \frac{\omega(1-r)}{1-r},
$$

where $\alpha_{3}=\left(\frac{n-1}{n}+2^{2 n}\right) 2^{n} \alpha_{1}$.

Case 4.2. $k=1$.

Again, (2.23) implies

$$
\frac{\partial}{\partial x_{1}} P_{h}\left(r e_{1}, \xi\right)=\frac{2(n-1)\left(1-\left|x_{0}\right|^{2}\right)^{n-1}\left(\xi_{1}-\left|x_{0}\right|\right)}{\left|x_{0}-\xi\right|^{2 n}}-\frac{2(n-1)\left(1-\left|x_{0}\right|^{2}\right)^{n-2}\left|x_{0}\right|}{\left|x_{0}-\xi\right|^{2 n-2}}
$$

and so

$$
\begin{aligned}
\left|\frac{\partial \Phi}{\partial x_{1}}\left(r e_{1}\right)\right|= & \left|\int_{\mathbb{S}^{n-1}} \frac{\partial}{\partial x_{1}} P_{h}\left(x_{0}, \xi\right)\left(\phi(\xi)-\phi\left(\xi_{0}\right)\right) d \sigma(\xi)\right| \\
\leq & 2(n-1)\left(1-\left|x_{0}\right|^{2}\right)^{n-1} \int_{\mathbb{S}^{n-1}} \frac{\omega\left(\left|\xi-\xi_{0}\right|\right)\left|\xi_{1}-\right| x_{0}||}{\left|x_{0}-\xi\right|^{2 n}} d \sigma(\xi) \\
& +2(n-1)\left(1-\left|x_{0}\right|^{2}\right)^{n-2}\left|x_{0}\right| \int_{\mathbb{S}^{n-1}} \frac{\omega\left(\left|\xi-\xi_{0}\right|\right)}{\left|x_{0}-\xi\right|^{2 n-2}} d \sigma(\xi)
\end{aligned}
$$

Since

$$
\left|\xi_{1}-\right| x_{0}|| \leq\left|\xi_{1}-1\right|+|1-| x_{0}|| \leq\left|\xi-\xi_{0}\right|+1-\left|x_{0}\right|,
$$

we get

$$
\begin{aligned}
\left|\frac{\partial \Phi}{\partial x_{1}}\left(r e_{1}\right)\right| \leq & 2(n-1)\left(1-\left|x_{0}\right|^{2}\right)^{n-1} \int_{\mathbb{S}^{n-1}} \frac{\omega\left(\left|\xi-\xi_{0}\right|\right)\left|\xi-\xi_{0}\right|}{\left|x_{0}-\xi\right|^{2 n}} d \sigma(\xi) \\
& +2(n-1)\left(1-\left|x_{0}\right|\right)^{n}\left(1+\left|x_{0}\right|\right)^{n-1} \int_{\mathbb{S}^{n-1}} \frac{\omega\left(\left|\xi-\xi_{0}\right|\right)}{\left|x_{0}-\xi\right|^{2 n}} d \sigma(\xi) \\
& +2(n-1)\left(1-\left|x_{0}\right|^{2}\right)^{n-2}\left|x_{0}\right| \int_{\mathbb{S}^{n-1}} \frac{\omega\left(\left|\xi-\xi_{0}\right|\right)}{\left|x_{0}-\xi\right|^{2 n-2}} d \sigma(\xi) .
\end{aligned}
$$


Furthermore, Lemma 4.2 guarantees that

$$
\begin{gathered}
\int_{\mathbb{S}^{n-1}} \frac{\omega\left(\left|\xi-\xi_{0}\right|\right)\left|\xi-\xi_{0}\right|}{\left|x_{0}-\xi\right|^{2 n}} d \sigma(\xi) \leq \alpha_{1} \frac{\omega(1-r)}{(1-r)^{n}}\left(\frac{1}{n}+\frac{4^{n}}{n-1}\right), \\
\int_{\mathbb{S}^{n-1}} \frac{\omega\left(\left|\xi-\xi_{0}\right|\right)}{\left|x_{0}-\xi\right|^{2 n}} d \sigma(\xi) \leq \alpha_{1} \frac{\omega(1-r)}{(1-r)^{n+1}}\left(\frac{1}{n-1}+\frac{4^{n}}{n}\right)
\end{gathered}
$$

and

$$
\int_{\mathbb{S}^{n-1}} \frac{\omega\left(\left|\xi-\xi_{0}\right|\right)}{\left|x_{0}-\xi\right|^{2 n-2}} d \sigma(\xi) \leq \alpha_{1} \frac{\omega(1-r)}{(1-r)^{n-1}}\left(\frac{1}{n-1}+\frac{4^{n-1}}{n-2}\right)
$$

Hence

$$
\left|\frac{\partial \Phi}{\partial x_{1}}\left(r e_{1}\right)\right| \leq \alpha_{4} \frac{\omega(1-r)}{1-r}
$$

where

$$
\alpha_{4}=(n-1)\left(\frac{2^{n}+8^{n}}{n}+\frac{2^{n}+8^{n}+2^{n-1}}{n-1}+\frac{8^{n-1}}{n-2}\right) \alpha_{1}
$$

By letting $\alpha_{2}=\max \left\{\alpha_{3}, \alpha_{4}\right\}$, we see that the lemma is true.

Now, we are ready to state and prove the main lemma in this section.

Lemma 4.4. Suppose $\phi$ and $\omega$ satisfy the conditions in Theorem 1.3. Let $\alpha_{0}=$ $\sqrt{n} \alpha_{2}$, where $n \geq 3$ and $\alpha_{2}=\alpha_{2}(n)$ is the same constant as in Lemma 4.3. Then

$$
\|D \Phi(x)\| \leq \alpha_{0} \frac{\omega(1-|x|)}{1-|x|}
$$

in $\mathbb{B}^{n}$.

Proof. Let $x_{0} \in \mathbb{B}^{n}$. We divide the proof into two cases.

Case 4.3. $x_{0} \in\left[0, e_{1}\right)$.

Since the Cauchy-Schwarz inequality implies

$$
\left\|D \Phi\left(x_{0}\right)\right\|=\sup _{\zeta \in \mathbb{S}^{n-1}}\left|D \Phi\left(x_{0}\right) \zeta\right|=\sup _{\zeta \in \mathbb{S}^{n-1}}\left|\sum_{k=1}^{n} \frac{\partial \Phi}{\partial x_{k}}\left(x_{0}\right) \cdot \zeta_{k}\right| \leq\left(\sum_{k=1}^{n}\left|\frac{\partial \Phi}{\partial x_{k}}\left(x_{0}\right)\right|^{2}\right)^{\frac{1}{2}}
$$

we see from Lemma 4.3 that

$$
\left\|D \Phi\left(x_{0}\right)\right\| \leq\left(\sum_{k=1}^{n}\left|\frac{\partial \Phi}{\partial x_{k}}\left(x_{0}\right)\right|^{2}\right)^{\frac{1}{2}} \leq \sqrt{n} \alpha_{2} \frac{\omega\left(1-\left|x_{0}\right|\right)}{1-\left|x_{0}\right|} .
$$

The proof of Lemma 4.4 holds in this case.

Case 4.4. $x_{0} \notin\left[0, e_{1}\right)$. 
For the proof in this case, we choose a unitary transformation $U$ such that $U\left(r e_{1}\right)=x_{0}, r=\left|x_{0}\right|$, and for $y \in \mathbb{B}^{n}$, let

$$
W(y)=: \Phi(U(y)) .
$$

By Theorem E, we see that

$$
W=P_{h}[\phi] \circ U=P_{h}[\phi \circ U] .
$$

Then we have the following claim.

Claim 4.3. $\left\|D W\left(r e_{1}\right)\right\| \leq \sqrt{n} \alpha_{2} \frac{\omega(1-r)}{1-r}$.

The assumption " $|\phi(\xi)-\phi(\eta)| \leq \omega(|\xi-\eta|)$ " implies that for $\xi, \eta \in \mathbb{S}^{n-1}$,

$$
|\phi(U(\xi))-\phi(U(\eta))| \leq \omega(|U(\xi)-U(\eta)|)=\omega(|\xi-\eta|) .
$$

Thus, by replacing $\Phi$ by $\Phi \circ U$, the similar reasoning as in the discussions of Case 4.3 shows that

$$
\left\|D W\left(r e_{1}\right)\right\| \leq \sqrt{n} \alpha_{2} \frac{\omega(1-r)}{1-r}
$$

which is what we want.

Now, we are ready to finish the proof of the lemma in this case. By applying the chain rule, we obtain

$\left.D W(y)\right|_{y=r e_{1}}=\left.D(\Phi \circ U)(y)\right|_{y=r e_{1}}=\left.(D \Phi) \circ U(y)\right|_{y=r e_{1}} \times\left. D U(y)\right|_{y=r e_{1}}=D \Phi\left(x_{0}\right) \times U$,

where $x$ denotes the usual matrix product. Then

$$
\left\|D \Phi\left(x_{0}\right)\right\|=\left\|D \Phi\left(x_{0}\right) \times U\right\|=\left\|D W\left(r e_{1}\right)\right\| \leq \sqrt{n} \alpha_{2}(n) \frac{\omega(1-r)}{1-r} .
$$

By (4.4) and (4.5), we complete the proof of Lemma 4.4.

Proof of Theorem 1.3. Now, we are ready to prove Theorem 1.3 by applying Lemma 4.4.

Lemma 4.1(a) implies that $\Phi \in C^{1}\left(\mathbb{B}^{n}\right)$ and so $\Phi$ is differentiable. Since $\mathbb{B}^{n}$ is a $\Lambda_{w}$-extension domain for a fast majorant $\omega$ (cf. [13, Section 1]), it follows from the mean-value theorem of differentials (see e.g. [32, Theorem 9.19]), (1.5) and Lemma 4.4 that there is a rectifiable curve $\gamma \subset \mathbb{B}^{n}$ joining $x$ to $y$ satisfying

$$
|\Phi(y)-\Phi(x)| \leq \int_{\gamma}\|D \Phi(\zeta)\| d s(\zeta) \leq \alpha_{0} \int_{\gamma} \frac{\omega\left(\delta_{\mathbb{B}^{n}}(\zeta)\right)}{\delta_{\mathbb{B}^{n}}(\zeta)} d s(\zeta) \leq C \alpha_{0} \omega(|x-y|),
$$

since $\delta_{\mathbb{B}^{n}}(\zeta)=1-|\zeta|$ for $\zeta \in \mathbb{B}^{n}$, where $C=C\left(\mathbb{B}^{n}, \omega\right)$ is the same constant as in (1.5). So the proof of this Theorem 1.3 is complete. 


\section{LipsChitz CONTINUITY OF $\Psi=G_{h}[\psi]$}

In this section, Theorem 1.4 is proved through a series of lemmas. From this and Theorem 1.3, we derive Lipschitz continuity of $u=P_{h}[\phi]-G_{h}[\psi]$, i.e. Theorem 1.2.

First, let us recall the following lemma from [30].

Theorem G. ([30, Section 2]) Let $f$ be a continuous function in $[-1,1]$. Then for any $\eta \in \mathbb{S}^{n-1}$ and $n \geq 3$,

$$
\int_{\mathbb{S}^{n-1}} f(\langle\xi, \eta\rangle) d \sigma(\xi)=\frac{\Gamma\left(\frac{n}{2}\right)}{\Gamma\left(\frac{n-1}{2}\right) \Gamma\left(\frac{1}{2}\right)} \int_{-1}^{1}\left(1-t^{2}\right)^{\frac{n-3}{2}} f(t) d t .
$$

Lemma 5.1. Let

$$
I_{1}(s)=: \int_{0}^{1} F\left(1, \frac{4-n}{2} ; \frac{n}{2} ; t s\right) d t .
$$

(1) If $n \geq 4$, then for $s \in[0,1)$,

$$
\left|I_{1}(s)\right| \leq \mu_{2,2}
$$

where $\mu_{2,2}=\mu_{2}(n, 1,4,1)$ is defined in Lemma 2.1;

(2) If $n=3$ and $s_{0} \in(0,1)$, then for $s \in\left[0, s_{0}\right]$,

$$
\left|I_{1}(s)\right| \leq \frac{1}{1-s_{0}} \text {. }
$$

Proof. It follows from Lemma 2.2 that

$$
I_{1}(s)=\sum_{k=0}^{\infty} \frac{(1)_{k}\left(\frac{4-n}{2}\right)_{k}}{\left(\frac{n}{2}\right)_{k} k !} \frac{s^{k}}{k+1} .
$$

If $n \geq 4$, the result follows from Lemma 2.1.

If $n=3$, then for any $s_{0} \in(0,1)$, we have

$$
I_{1}(s)=\sum_{k=0}^{\infty} \frac{(1)_{k}\left(\frac{4-n}{2}\right)_{k}}{\left(\frac{n}{2}\right)_{k} k !} \frac{s^{k}}{k+1} \leq \sum_{k=0}^{\infty} s^{k} \leq \frac{1}{1-s_{0}} .
$$

Hence Lemma 5.1 is proved.

By Lemma 5.1, we have the following estimate.

Lemma 5.2. If $n \geq 3$, then for all $x \in \mathbb{B}^{n}$,

$$
J_{n}(x)=: \int_{\mathbb{B}^{n}} \frac{d \nu(y)}{|y|^{n-2}[x, y]^{2}} \leq \mu_{5},
$$

where $\mu_{5}=\max \left\{\frac{n}{2} \mu_{2,2}, 65 \frac{7}{8}\right\}$, where $\mu_{2,2}$ is the same constant as in Lemma 5.1.

Proof. For $n \geq 3$, (2.4) leads to

$$
J_{n}(x)=\int_{\mathbb{B}^{n}} \frac{d \nu(y)}{|y|^{n-2}[x, y]^{2}}=n \int_{0}^{1} \rho d \rho \int_{\mathbb{S}^{n-1}} \frac{d \sigma(\xi)}{[x, \rho \xi]^{2}} .
$$


By (2.10), we have

$$
\int_{\mathbb{S}^{n-1}} \frac{d \sigma(\xi)}{[x, \rho \xi]^{2}}=\int_{\mathbb{S}^{n-1}}\left(1+\rho^{2}|x|^{2}-2 \rho|x|\left\langle\frac{x}{|x|}, \xi\right\rangle\right)^{-1} d \sigma(\xi),
$$

and so Theorem G and (2.6) lead to

$$
\begin{aligned}
\int_{\mathbb{S}^{n-1}} \frac{d \sigma(\xi)}{[x, \rho \xi]^{2}} & =\frac{\Gamma\left(\frac{n}{2}\right)}{\Gamma\left(\frac{n-1}{2}\right) \Gamma\left(\frac{1}{2}\right)} \int_{-1}^{1}\left(1-t^{2}\right)^{\frac{n-3}{2}}\left(1+\rho^{2}|x|^{2}-2 \rho|x| t\right)^{-1} d t \\
& =F\left(1, \frac{4-n}{2} ; \frac{n}{2} ; \rho^{2}|x|^{2}\right) .
\end{aligned}
$$

Hence we have

$$
J_{n}(x)=n \int_{0}^{1} \rho F\left(1, \frac{4-n}{2} ; \frac{n}{2} ; \rho^{2}|x|^{2}\right) d \rho .
$$

When $n \geq 4$, it follows from Lemma 5.1 that for all $x \in \mathbb{B}^{n}$,

$$
J_{n}(x) \leq \frac{n}{2} \mu_{2,2} .
$$

In the following, we assume that $n=3$. Then we have the following assertion.

Claim 5.1. $J_{3}(x) \leq 65 \frac{7}{8}$.

We divide the proof into two cases according to the value of $|x|$.

Case 5.1. $\frac{3}{4} \leq|x|<1$.

Since $n=3$, by (2.10), we see that

$$
J_{3}(x)=\int_{\mathbb{B}^{3}} \frac{d \nu(y)}{|y|[x, y]^{2}} \leq \int_{\mathbb{B}^{3}} \frac{d \nu(y)}{|y| \cdot|y-x|^{2}} .
$$

Let $\delta_{1}=|x| / 3$. By (2.4) and elementary calculations, we have that

$$
\begin{gathered}
\int_{\mathbb{B}^{3}\left(0, \delta_{1}\right)} \frac{d \nu(y)}{|y| \cdot|y-x|^{2}} \leq \int_{\mathbb{B}^{3}\left(0, \delta_{1}\right)} \frac{d \nu(y)}{4|y| \delta_{1}^{2}}=\frac{3}{8}, \\
\int_{\mathbb{B}^{3} \cap \mathbb{B}^{3}\left(x, \delta_{1}\right)} \frac{d \nu(y)}{|y| \cdot|y-x|^{2}} \leq \int_{\mathbb{B}^{3}\left(x, \delta_{1}\right)} \frac{d \nu(y)}{2|y-x|^{2} \delta_{1}}=\int_{\mathbb{B}^{3}\left(0, \delta_{1}\right)} \frac{d \nu(y)}{2|y|^{2} \delta_{1}}=\frac{3}{2}
\end{gathered}
$$

and

$$
\int_{\mathbb{B}^{3} \backslash\left(\mathbb{B}^{3}\left(0, \delta_{1}\right) \cup \mathbb{B}^{3}\left(x, \delta_{1}\right)\right)} \frac{d \nu(y)}{|y| \cdot|y-x|^{2}} \leq \int_{\mathbb{B}^{3} \backslash\left(\mathbb{B}^{3}\left(0, \delta_{1}\right) \cup \mathbb{B}^{3}\left(x, \delta_{1}\right)\right)} \frac{d \nu(y)}{\delta_{1}^{3}} \leq \frac{1}{\delta_{1}^{3}} \leq 64 .
$$

These inequalities show that the claim holds since

$$
\mathbb{B}^{3}=\mathbb{B}^{3}\left(0, \delta_{1}\right) \cup \mathbb{B}^{3}\left(x, \delta_{1}\right) \cup\left(\mathbb{B}^{3} \backslash\left(\mathbb{B}^{3}\left(0, \delta_{1}\right) \cup \mathbb{B}^{3}\left(x, \delta_{1}\right)\right)\right) .
$$

Case 5.2. $|x|<\frac{3}{4}$. 
Under this assumption, we see from (5.1) and Lemma 5.1 that

$$
J_{3}(x)=\frac{3}{2} \int_{0}^{1} F\left(1, \frac{1}{2} ; \frac{3}{2} ; \rho^{2}|x|^{2}\right) d\left(\rho^{2}\right) \leq \frac{3}{2} \cdot \frac{1}{1-\frac{9}{16}}=\frac{24}{7},
$$

as required. So Claim 5.1 is proved.

Now, we obtain from (5.2) and Claim 5.1 that for all $x \in \mathbb{B}^{n}$,

$$
J_{n}(x)=\int_{\mathbb{B}^{n}} \frac{d \nu(u)}{|u|^{n-2}[x, u]^{2}} \leq \max \left\{\frac{n}{2} \mu_{2,2}, 65 \frac{7}{8}\right\}
$$

and hence the proof of Lemma 5.2 is complete.

Based on Theorem G, Lemmas 5.1 and 5.2, we obtain some properties of the two unbounded integrals $G_{h}[\psi](x)$ and

$$
\int_{\mathbb{B}^{n}} \frac{\partial G_{h}}{\partial x_{k}}(x, y) \psi(y) d \tau(y),
$$

which will be presented in the next four lemmas. The first two lemmas deal with the uniform convergence of these two integrals, respectively.

Lemma 5.3. Suppose $n \geq 3, \psi \in C\left(\mathbb{B}^{n}, \mathbb{R}^{n}\right)$ and $|\psi(x)| \leq M\left(1-|x|^{2}\right)$ in $\mathbb{B}^{n}$, where $M$ is a constant. Then for all $0<r_{0}<1$, the unbounded integral $G_{h}[\psi](x)$ is uniformly convergent w.r.t. $x$ in $\overline{\mathbb{B}^{n}}\left(0, r_{0}\right)$.

Proof. By the assumption " $|\psi(x)| \leq M\left(1-|x|^{2}\right)$ ", we see from (2.25) that

$$
\left|G_{h}[\psi](x)\right| \leq \frac{M}{n} \int_{\mathbb{B}^{n}}\left[\frac{1}{\left(1-|y|^{2}\right)^{n-1}} \int_{\left|\varphi_{x}(y)\right|}^{1} \frac{\left(1-s^{2}\right)^{n-2}}{s^{n-1}} d s\right] d \nu(y) .
$$

For $x \in \overline{\mathbb{B}^{n}}\left(0, r_{0}\right),(2.11)$ leads to

$$
[x, y] \geq 1-|x| \geq 1-r_{0} .
$$

Since

$$
\int_{\mathbb{B}^{n}}\left[\frac{1}{\left(1-|y|^{2}\right)^{n-1}} \int_{\left|\varphi_{x}(y)\right|}^{1} \frac{\left(1-s^{2}\right)^{n-2}}{s^{n-1}} d s\right] d \nu(y)=\int_{\mathbb{B}^{n}} \frac{g\left(\left|\varphi_{x}(y)\right|\right)}{\left(1-|y|^{2}\right)^{n-1}} d \nu(y),
$$

we see that

$$
\begin{aligned}
& \int_{\mathbb{B}^{n}}\left[\frac{1}{\left(1-|y|^{2}\right)^{n-1}} \int_{\left|\varphi_{x}(y)\right|}^{1} \frac{\left(1-s^{2}\right)^{n-2}}{s^{n-1}} d s\right] d \nu(y) \\
\leq & \frac{1}{n(n-2)} \int_{\mathbb{B}^{n}} \frac{\left(1-\left|\varphi_{x}(y)\right|^{2}\right)^{n-1}}{\left|\varphi_{x}(y)\right|^{n-2}\left(1-|y|^{2}\right)^{n-1}} d \nu(y) \\
< & \frac{1}{n(n-2)} \int_{\mathbb{B}^{n}} \frac{1}{[x, y]^{n}|x-y|^{n-2}} d \nu(y) \\
\leq & \frac{1}{n(n-2)\left(1-r_{0}\right)^{n}} \int_{\mathbb{B}^{n}} \frac{1}{|x-y|^{n-2}} d \nu(y) .
\end{aligned}
$$


Thus in order to prove the uniform convergence of $G_{h}[\psi](x)$ in $\overline{\mathbb{B}^{n}}\left(0, r_{0}\right)$, we only need to prove that

$$
F_{n-2}(x)=\int_{\mathbb{B}^{n}} \frac{1}{|x-y|^{n-2}} d \nu(y)
$$

is uniformly convergent. In fact, we shall prove the following more general result.

Claim 5.2. The integral $F_{k}(x)=\int_{\mathbb{B}^{n}} \frac{1}{|x-y|^{k}} d \nu(y)$ is uniformly convergent w.r.t. $x$ in $\overline{\mathbb{B}^{n}}\left(0, r_{0}\right)$, where $1 \leq k \leq n-1$ and $0<r_{0}<1$.

Let $\delta_{2}=\frac{1-r_{0}}{3}$. Then

$$
\mathbb{B}^{n}=\mathbb{B}^{n}\left(x, \delta_{2}\right) \cup\left(\mathbb{B}^{n} \backslash \mathbb{B}^{n}\left(x, \delta_{2}\right)\right) \text { and } \mathbb{B}^{n}\left(x, \delta_{2}\right) \subset \mathbb{B}^{n} .
$$

Hence

$$
F_{k}(x)=F_{k, 1}(x)+F_{k, 2}(x),
$$

where

$$
F_{k, 1}(x)=\int_{\mathbb{B}^{n} \backslash \mathbb{B}^{n}\left(x, \delta_{2}\right)} \frac{1}{|x-y|^{k}} d \nu(y) \text { and } F_{k, 2}(x)=\int_{\mathbb{B}^{n}\left(x, \delta_{2}\right)} \frac{1}{|x-y|^{k}} d \nu(y) .
$$

Subclaim 1. $F_{k, 1}(x)$ and $F_{k, 2}(x)$ are uniformly convergent w.r.t. $x$ in $\overline{\mathbb{B}^{n}}\left(0, r_{0}\right)$, where $1 \leq k \leq n-1$ and $0<r_{0}<1$.

Since for all $y \in \mathbb{B}^{n} \backslash \mathbb{B}^{n}\left(x, \delta_{2}\right)$,

$$
\frac{1}{|x-y|^{k}} \leq \frac{1}{\delta_{2}^{k}}
$$

by the Weierstrass test for uniform convergence, the uniform convergence of $F_{k, 1}(x)$ in $\overline{\mathbb{B}^{n}}\left(0, r_{0}\right)$ is obvious.

For any $0<\delta \leq \delta_{2}$, let $y=x+w$. Then it follows from (2.4) that

$$
\int_{\mathbb{B}^{n}(0, \delta)} \frac{1}{|w|^{k}} d \nu(w)=\frac{n}{n-k} \delta^{n-k} \leq n \delta .
$$

By definition, we easily know that $F_{k, 2}(x)$ is uniformly convergent w.r.t. $x$ in $\overline{\mathbb{B}^{n}}\left(0, r_{0}\right)$. Hence Subclaim 1 is proved.

Subclaim 1 implies the uniform convergence of $F_{k}(x)$ in $\overline{\mathbb{B}^{n}}\left(0, r_{0}\right)$, and thus the proof of Claim 5.2 is complete.

Let $k=n-2$. Then by Claim 5.2 , we know that $G_{h}[\psi](x)$ is also uniformly convergent in $\overline{\mathbb{B}^{n}}\left(0, r_{0}\right)$, and so the lemma is proved.

Now, we are going to prove the first main lemma for the proof of Theorem 1.4.

Lemma 5.4. Suppose $n \geq 3, k \in\{1, \ldots, n\}, \psi \in C\left(\mathbb{B}^{n}, \mathbb{R}^{n}\right)$ and $|\psi(x)| \leq M(1-$ $\left.|x|^{2}\right)$ in $\mathbb{B}^{n}$, where $M$ is a constant. Then

(1) for $0<r_{0}<1$, the unbounded integral

$$
I_{2, k}(x)=: \int_{\mathbb{B}^{n}}\left|\frac{\partial}{\partial x_{k}} G_{h}(x, y) \psi(y)\right| d \tau(y)
$$

is uniformly convergent w.r.t. $x$ in $\overline{\mathbb{B}^{n}}\left(0, r_{0}\right)$; 
(2) for all $x \in \mathbb{B}^{n}$, there exists a constant $\beta_{1}=\beta_{1}(n, M)$ such that

$$
I_{2, k}(x) \leq \beta_{1} .
$$

Proof. First, we easily see from (2.10), (2.12), (2.14) and (2.19) that for $x \neq y$,

$$
\begin{aligned}
\frac{\partial}{\partial x_{k}} G_{h}(x, y)= & -\frac{\left(x_{k}-y_{k}\right)\left(1-|x|^{2}\right)^{n-1}\left(1-|y|^{2}\right)^{n-1}}{n|x-y|^{n}[x, y]^{n}} \\
& -\frac{x_{k}\left(1-|x|^{2}\right)^{n-2}\left(1-|y|^{2}\right)^{n-1}}{n|x-y|^{n-2}[x, y]^{n}} .
\end{aligned}
$$

Then (2.11) implies that for $x \in \mathbb{B}^{n}\left(0, r_{0}\right),[x, y] \geq 1-r_{0}$, and hence

$$
\begin{aligned}
\left|\frac{\partial}{\partial x_{k}} G_{h}(x, y)\right| & \leq \frac{\left(1-|x|^{2}\right)^{n-1}\left(1-|y|^{2}\right)^{n-1}}{n|x-y|^{n-1}[x, y]^{n}}+\frac{\left(1-|x|^{2}\right)^{n-2}\left(1-|y|^{2}\right)^{n-1}}{n|x-y|^{n-2}[x, y]^{n}} \\
& \leq \frac{\left(1-|y|^{2}\right)^{n-1}}{n\left(1-r_{0}\right)^{n}}\left(\frac{1}{|x-y|^{n-1}}+\frac{1}{|x-y|^{n-2}}\right) .
\end{aligned}
$$

Thus the assumption " $|\psi(x)| \leq M\left(1-|x|^{2}\right)$ " implies that for all $x \in \mathbb{B}^{n}\left(0, r_{0}\right)$,

$$
\begin{aligned}
I_{2, k}(x) & \leq M \int_{\mathbb{B}^{n}}\left|\frac{\partial}{\partial x_{k}} G_{h}(x, y)\right|\left(1-|y|^{2}\right) d \tau(y) \\
& \leq \frac{M}{n\left(1-r_{0}\right)^{n}} \int_{\mathbb{B}^{n}}\left(\frac{1}{|x-y|^{n-1}}+\frac{1}{|x-y|^{n-2}}\right) d \nu(y) .
\end{aligned}
$$

The uniform convergence of $I_{2, k}(x)$ w.r.t. $x$ in $\overline{\mathbb{B}^{n}}\left(0, r_{0}\right)$ follows from Claim 5.2, and thus Lemma 5.4(1) holds.

Next, we prove Lemma 5.4(2). It follows from (5.3) that

$$
I_{2, k}(x) \leq \frac{1}{n}\left(I_{3, k}(x)+I_{4, k}(x)\right),
$$

where

$$
I_{3, k}(x)=\int_{\mathbb{B}^{n}} \frac{\left|x_{k}-y_{k}\right|\left(1-|x|^{2}\right)^{n-1}}{|x-y|^{n}[x, y]^{n}\left(1-|y|^{2}\right)}|\psi(y)| d \nu(y)
$$

and

$$
I_{4, k}(x)=\int_{\mathbb{B}^{n}} \frac{\left|x_{k}\right|\left(1-|x|^{2}\right)^{n-2}}{|x-y|^{n-2}[x, y]^{n}\left(1-|y|^{2}\right)}|\psi(y)| d \nu(y) .
$$

Next, we estimate $\left|I_{3, k}(x)\right|$ and $\left|I_{4, k}(x)\right|$, respectively.

Claim 5.3. For $x \in \mathbb{B}^{n}$,

$$
\left|I_{3, k}(x)\right| \leq \frac{n M}{2} \mu_{2,3},
$$

where $\mu_{2,3}=\mu_{2}\left(n, \frac{1}{2}, 3, \frac{1}{2}\right)$ is the same constant as in Lemma 2.1. 
Let $y=\varphi_{x}(w)$. Then the equalities (2.4), (2.13), (2.15) and the assumption " $|\psi(x)| \leq M\left(1-|x|^{2}\right)$ " imply that

$$
\begin{aligned}
\left|I_{3, k}(x)\right| & \leq M \int_{\mathbb{B}^{n}} \frac{\left(1-|x|^{2}\right)^{n-1}|x-y|}{|x-y|^{n}[x, y]^{n}} d \nu(y) \\
& =M \int_{\mathbb{B}^{n}} \frac{\left(1-|x|^{2}\right)^{n-1} J_{\varphi_{x}}(w)}{\left|x-\varphi_{x}(w)\right|^{n-1}\left[x, \varphi_{x}(w)\right]^{n}} d \nu(w) \\
& =M \int_{\mathbb{B}^{n}} \frac{d \nu(w)}{[x, w] \cdot|w|^{n-1}} \\
& =n M \int_{0}^{1} \int_{\mathbb{S}^{n-1}} \frac{d \sigma(\xi)}{[x, \rho \xi]} d \rho .
\end{aligned}
$$

Moreover, by (2.10), we have

$$
\int_{\mathbb{S}^{n-1}} \frac{d \sigma(\xi)}{[x, \rho \xi]}=\int_{\mathbb{S}^{n-1}}\left(1+\rho^{2}|x|^{2}-2 \rho|x|\left\langle\frac{x}{|x|}, \xi\right\rangle\right)^{-\frac{1}{2}} d \sigma(\xi),
$$

which, together with Theorem G and (2.6), implies that

$$
\begin{aligned}
\int_{\mathbb{S}^{n-1}} \frac{d \sigma(\xi)}{[x, \rho \xi]} & =\frac{\Gamma\left(\frac{n}{2}\right)}{\Gamma\left(\frac{n-1}{2}\right) \Gamma\left(\frac{1}{2}\right)} \int_{-1}^{1}\left(1-s^{2}\right)^{\frac{n-3}{2}}\left(1+\rho^{2}|x|^{2}-2 \rho|x| s\right)^{-\frac{1}{2}} d s \\
& =F\left(\frac{1}{2}, \frac{3-n}{2} ; \frac{n}{2} ; \rho^{2}|x|^{2}\right) .
\end{aligned}
$$

Hence Lemma 2.3 leads to

$$
\left|I_{3, k}(x)\right| \leq n M \int_{0}^{1} F\left(\frac{1}{2}, \frac{3-n}{2} ; \frac{n}{2} ; \rho^{2}|x|^{2}\right) d \rho \leq \frac{n M}{2} \mu_{2,3},
$$

as required, where $\mu_{2,3}=\mu_{2}\left(n, \frac{1}{2}, 3, \frac{1}{2}\right)$.

Claim 5.4. For $x \in \mathbb{B}^{n}$, we have

$$
\left|I_{4, k}(x)\right| \leq M \mu_{5}
$$

where $\mu_{5}=\mu_{5}(n)$ is the same constant as in Lemma 5.2.

Obviously, the assumption " $|\psi(x)| \leq M\left(1-|x|^{2}\right)$ " implies that

$$
\left|I_{4, k}(x)\right| \leq M \int_{\mathbb{B}^{n}} \frac{\left(1-|x|^{2}\right)^{n-2}}{|x-y|^{n-2}[x, y]^{n}} d \nu(y) .
$$

Let $y=\varphi_{x}(w)$. By (2.13), (2.15) and Lemma 5.2, we get

$$
\begin{aligned}
\left|I_{4, k}\right| & \left.\leq M \int_{\mathbb{B}^{n}} \frac{\left(1-|x|^{2}\right)^{n-2} J_{\varphi_{x}}(w)}{\left|x-\varphi_{x}(w)\right|^{n-2}\left[x, \varphi_{x}(w)\right]^{n}} d \nu(w) \quad \text { (substituting } y=\varphi_{x}(w)\right) \\
& =M \int_{\mathbb{B}^{n}} \frac{d \nu(w)}{|w|^{n-2}[x, w]^{2}} \leq M \mu_{5}(n) .
\end{aligned}
$$

By taking $\beta_{1}=\frac{M}{2} \mu_{2,3}+\frac{M}{n} \mu_{5}$, we see that Lemma 5.4(2) holds, and so the proof of the lemma is finished. 
Lemma 5.5. Suppose $n \geq 3, \psi \in C\left(\mathbb{B}^{n}, \mathbb{R}^{n}\right)$ and $|\psi(x)| \leq M\left(1-|x|^{2}\right)$ in $\mathbb{B}^{n}$, where $M$ is a constant. Then for all $0<r_{0}<1$ and $k \in\{1,2, \ldots, n\}$,

$$
G_{h}[\psi](x) \text { and } \int_{\mathbb{B}^{n}} \frac{\partial}{\partial x_{k}} G_{h}(x, y) \psi(y) d \tau(y)
$$

are continuous in $\overline{\mathbb{B}^{n}}\left(0, r_{0}\right)$, respectively.

Proof. In order to check the continuity of $G_{h}[\psi](x)$ in $\overline{\mathbb{B}^{n}}\left(0, r_{0}\right)$, we only need to prove that $G_{h}[\psi](x)$ is continuous at every fixed point $x_{0} \in \overline{\mathbb{B}^{n}}\left(0, r_{0}\right)$. Assume that $x_{0} \in \overline{\mathbb{B}^{n}}\left(0, r_{0}\right)$ and $x_{0}+\Delta x \in \overline{\mathbb{B}^{n}}\left(0, r_{0}\right)$.

By Lemma 5.3, we see that $G_{h}[\psi](x)$ is uniformly convergent in $\overline{\mathbb{B}^{n}}\left(0, r_{0}\right)$. Then for any $\varepsilon_{1}>0$, there exist constants $\iota_{1}=\iota_{1}\left(\varepsilon_{1}\right) \rightarrow 1^{-}$and $\iota_{2}=\iota_{2}\left(\varepsilon_{1}\right) \rightarrow 0^{+}$such that for any $x \in \overline{\mathbb{B}^{n}}\left(0, r_{0}\right)$,

$$
\begin{gathered}
\mathbb{B}^{n}\left(x, \iota_{2}\right) \subset \mathbb{B}^{n}\left(0, \iota_{1}\right), \\
\left|\int_{\mathbb{B}^{n} \backslash \mathbb{B}^{n}\left(0, \iota_{1}\right)} G_{h}(x, y) \psi(y) d \tau(y)\right|<\varepsilon_{1} \text { and }\left|\int_{\mathbb{B}^{n}\left(x, \iota_{2}\right)} G_{h}(x, y) \psi(y) d \tau(y)\right|<\varepsilon_{1} .
\end{gathered}
$$

Then

$$
\begin{aligned}
& \left|G_{h}[\psi]\left(x_{0}+\Delta x\right)-G_{h}[\psi]\left(x_{0}\right)\right| \\
= & \mid \int_{\overline{\mathbb{B}^{n}}\left(0, \iota_{1}\right) \backslash \mathbb{B}^{n}\left(x_{0}, \iota_{2}\right)}\left[G_{h}\left(x_{0}+\Delta x, y\right)-G_{h}\left(x_{0}, y\right)\right] \psi(y) d \tau(y) \\
& +\int_{\mathbb{B}^{n}\left(x_{0}, \iota_{2}\right)}\left[G_{h}\left(x_{0}+\Delta x, y\right)-G_{h}\left(x_{0}, y\right)\right] \psi(y) d \tau(y) \\
& +\int_{\mathbb{B}^{n} \backslash \mathbb{B}^{n}\left(0, \iota_{1}\right)}\left[G_{h}\left(x_{0}+\Delta x, y\right)-G_{h}\left(x_{0}, y\right)\right] \psi(y) d \tau(y) \mid \\
\leq & \left|\int_{\mathbb{B}^{n}\left(0, \iota_{1}\right) \backslash \mathbb{B}^{n}\left(x_{0}, \iota_{2}\right)}\left[G_{h}\left(x_{0}+\Delta x, y\right)-G_{h}\left(x_{0}, y\right)\right] \frac{\psi(y)}{\left(1-|y|^{2}\right)^{n}} d \nu(y)\right|+4 \varepsilon_{1} .
\end{aligned}
$$

By (2.19), it is easy to see that the map $(x, y) \rightarrow G_{h}(x, y) \frac{\psi(y)}{\left(1-|y|^{2}\right)^{n}}$ is continuous (also uniformly continuous) on $\overline{\mathbb{B}^{n}}\left(x_{0}, \frac{1}{2} \iota_{2}\right) \times\left(\overline{\mathbb{B}^{n}}\left(0, \iota_{1}\right) \backslash \mathbb{B}^{n}\left(x_{0}, \iota_{2}\right)\right)$. Therefore, there exists $\iota^{\prime}=\iota^{\prime}\left(\varepsilon_{1}\right)<\frac{1}{2} \iota_{2}$ such that for all $|\Delta x|<\iota^{\prime}$ and for all $y \in \overline{\mathbb{B}^{n}}\left(0, \iota_{1}\right) \backslash \mathbb{B}^{n}\left(x_{0}, \iota_{2}\right)$,

$$
\left|\left(G_{h}\left(x_{0}+\Delta x, y\right)-G_{h}\left(x_{0}, y\right)\right) \frac{\psi(y)}{\left(1-|y|^{2}\right)^{n}}\right|<\varepsilon_{1} \text {. }
$$

Thus it follows from (5.4) and (5.5) that

$$
\left|G_{h}[\psi]\left(x_{0}+\Delta x\right)-G_{h}[\psi]\left(x_{0}\right)\right| \leq 5 \varepsilon_{1},
$$

which means that $G_{h}[\psi]$ is continuous at $x_{0}$. Hence the arbitrariness of $x_{0}$ shows that $G_{h}[\psi](x)$ is continuous in $\overline{\mathbb{B}^{n}}\left(0, r_{0}\right)$.

By applying Lemma 5.4, the continuity of

$$
\int_{\mathbb{B}^{n}} \frac{\partial}{\partial x_{k}} G_{h}(x, y) \psi(y) d \tau(y)
$$


in $\overline{\mathbb{B}^{n}}\left(0, r_{0}\right)$ can be proved in a similar way as above, where $k \in\{1, \ldots, n\}$. So the proof of this lemma is complete.

The following property is the second main lemma for the proof of Theorem 1.4.

Lemma 5.6. Suppose $n \geq 3, \psi \in C\left(\mathbb{B}^{n}, \mathbb{R}^{n}\right)$ and $|\psi(x)| \leq M\left(1-|x|^{2}\right)$ in $\mathbb{B}^{n}$, where $M$ is a constant. Then for all $x \in \mathbb{B}^{n}$ and $k \in\{1,2, \ldots, n\}$,

$$
\frac{\partial \Psi}{\partial x_{k}}(x)=\int_{\mathbb{B}^{n}} \frac{\partial}{\partial x_{k}} G_{h}(x, y) \psi(y) d \tau(y) .
$$

Proof. For all $x \in \mathbb{B}^{n}$, by Lemma 5.4 , we see that

$$
\int_{0}^{x_{k}} \int_{\mathbb{B}^{n}}\left|\frac{\partial}{\partial x_{k}} G_{h}(x, y) \frac{\psi(y)}{\left(1-|y|^{2}\right)^{n-1}}\right| d \nu(y) d x_{k} \leq \beta_{1},
$$

where $k \in\{1, \ldots, n\}$. It follows from Fubini's theorem [33, p. 165] that

$$
\int_{0}^{x_{k}} \int_{\mathbb{B}^{n}} \frac{\partial}{\partial x_{k}} G_{h}(x, y) \psi(y) d \tau(y) d x_{k}=\int_{\mathbb{B}^{n}} \int_{0}^{x_{k}} \frac{\partial}{\partial x_{k}} G_{h}(x, y) \frac{\psi(y)}{\left(1-|y|^{2}\right)^{n}} d x_{k} d \nu(y),
$$

which means

$$
\begin{aligned}
& \int_{0}^{x_{k}} \int_{\mathbb{B}^{n}} \frac{\partial}{\partial x_{k}} G_{h}(x, y) \psi(y) d \tau(y) d x_{k} \\
= & \int_{\mathbb{B}^{n}} \frac{G_{h}(x, y)}{\left(1-|y|^{2}\right)^{n}} \psi(y) d \nu(y)-\int_{\mathbb{B}^{n}} \frac{G_{h}\left(x_{k, 0}, y\right)}{\left(1-|y|^{2}\right)^{n}} \psi(y) d \nu(y),
\end{aligned}
$$

where $x_{k, 0}=\left(x_{1}, \ldots, x_{k-1}, 0, x_{k+1}, \ldots, x_{n}\right)$. Since $\int_{\mathbb{B}^{n}} \frac{\partial}{\partial x_{k}} G_{h}(x, y) \psi(y) d \tau(y)$ is continuous in $\overline{\mathbb{B}^{n}}\left(0, r_{0}\right)$, by differentiating w.r.t. $x_{k}$, we get

$$
\int_{\mathbb{B}^{n}} \frac{\partial}{\partial x_{k}} G_{h}(x, y) \psi(y) d \tau(y)=\frac{\partial}{\partial x_{k}} \int_{\mathbb{B}^{n}} \frac{G_{h}(x, y)}{\left(1-|y|^{2}\right)^{n}} \psi(y) d \nu(y) .
$$

Hence the proof of this lemma is finished.

Proof of Theorem 1.4. Lemmas 5.4(2), 5.6 and Cauchy-Schwarz inequality imply that

$$
\|D \Psi(x)\|=\sup _{\xi \in \mathbb{S}^{n-1}}|D \Psi(x) \xi| \leq\left(\sum_{k=1}^{n}\left|\frac{\partial \Psi}{\partial x_{k}}(x)\right|^{2}\right)^{\frac{1}{2}} \leq \sqrt{n} \beta_{1}=: \beta_{0},
$$

where $\beta_{1}=\beta_{1}(n, M)$ is the same constant as in Lemma 5.4(2). For any $x, y \in \mathbb{B}^{n}$, let $\gamma_{[x, y]}$ denote the segment between $x$ and $y$. By Lemmas 5.5 and 5.6, we know that $\Psi \in C^{1}\left(\mathbb{B}^{n}\right)$, and hence $\Psi$ is differentiable. Then the mean-value theorem of differentials leads to

$$
|\Psi(x)-\Psi(y)| \leq \beta_{0}|x-y|
$$

The proof of Theorem 1.4 is finished.

Based on Theorems 1.3 and 1.4, we are going to prove Theorem 1.2. 
Proof of Theorem 1.2. In this subsection, we always regard a point $x=\left(x_{1}, \ldots, x_{n}\right)$ in $\mathbb{R}^{n}$ as a column vector, for purposes of computing matrix products (which have been denoted by $x$ ).

For any $x, y \in \mathbb{B}^{n}$, by letting $\omega(t)=L t$ in Lemma 4.4, we obtain that for $x \in \mathbb{B}^{n}$,

$$
\|D \Phi(x)\| \leq L \alpha_{0}
$$

where $L$ is the same constant as in Theorem 1.2. It follows from the mean-value theorem of differentials that

$$
|\Phi(x)-\Phi(y)| \leq L \alpha_{0}|x-y|,
$$

and so Theorem 1.4 gives

$$
|u(x)-u(y)| \leq|\Phi(x)-\Phi(y)|+|\Psi(x)-\Psi(y)| \leq\left(L \alpha_{0}+\beta_{0}\right)|x-y| .
$$

Let $N=L \alpha_{0}+\beta_{0}$. Then the proof of Theorem 1.2 is complete.

\section{EXAMPLES}

In this section, we will first construct an example to show that the requirement $n \geq 3$ in Theorem 1.2 is necessary.

Example 6.1. Let $w_{0}\left(r e^{i \theta}\right)=\sum_{k=1}^{\infty} \frac{r^{k}}{k^{2}} \cos (k \theta)-\frac{M}{4}\left(1-r^{2}\right)$ in $\mathbb{D}$, where $M$ is a non-negative constant. Then,

(1) $w_{0} \in C^{2}(\mathbb{D}, \mathbb{R}) \cap C(\overline{\mathbb{D}}, \mathbb{R})$ and $\Delta_{h} w_{0}=M\left(1-|z|^{2}\right)^{2}$;

(2) $w_{0}$ is not Lipschitz continuous in $\mathbb{D}$;

(3) $\left.w_{0}\right|_{\mathbb{S}^{1}}$ is Lipschitz continuous in $\mathbb{S}^{1}$.

Proof. To prove that the function $w_{0}$ has the desired properties, let

$$
f(z)=\sum_{k=1}^{\infty} \frac{z^{k}}{k^{2}}
$$

Then for $z \in \mathbb{D}$,

$$
w_{0}(z)=\operatorname{Re} f(z)-\frac{M}{4}\left(1-|z|^{2}\right) .
$$

Obviously, $\operatorname{Re} f=P\left[\phi_{0}\right]$ is harmonic in $\mathbb{D}$, where $\phi_{0}\left(e^{i \theta}\right)=\sum_{k=1}^{\infty} \frac{1}{k^{2}} \cos (k \theta)$ is continuous in $\mathbb{S}^{1}$, and thus $\operatorname{Re} f \in C^{2}(\mathbb{D}, \mathbb{R}) \cap(\overline{\mathbb{D}}, \mathbb{R})$. By elementary computations, we see that $\Delta w_{0}=M$. Hence the first assertion in the example holds.

Since

$$
\frac{\partial}{\partial z} w_{0}(z)=\frac{1}{2} \sum_{k=1}^{\infty} \frac{z^{k-1}}{k}+\frac{M}{4} \bar{z}=-\frac{\log (1-z)}{2 z}+\frac{M}{4} \bar{z}
$$

and

$$
\frac{\partial}{\partial \bar{z}} w_{0}(z)=\frac{1}{2} \sum_{k=1}^{\infty} \frac{\bar{z}^{k-1}}{k}+\frac{M}{4} z=-\frac{\log (1-\bar{z})}{2 \bar{z}}+\frac{M}{4} z
$$

we easily see that

is unbounded in $\mathbb{D}$.

$$
\left\|D w_{0}(z)\right\|=\left|\frac{\partial}{\partial z} w_{0}(z)\right|+\left|\frac{\partial}{\partial \bar{z}} w_{0}(z)\right|
$$


Claim 6.1. The function $w_{0}$ is Lipschitz continuous if and only if $\left\|D w_{0}\right\|$ is bounded.

For the proof, we let $\partial_{\theta} w_{0}(z)$ denote the directional derivative of $w_{0}$. If $w_{0}$ is Lipschitz continuous with Lipschitz constant $L_{1}$, then

$$
\left|\partial_{\theta} w_{0}(z)\right|=\left|\lim _{r \rightarrow 0} \frac{w_{0}\left(z+r e^{i \theta}\right)-w_{0}(z)}{r}\right|=\lim _{r \rightarrow 0} \frac{\left|w_{0}\left(z+r e^{i \theta}\right)-w_{0}(z)\right|}{r} \leq L_{1} .
$$

Hence it follows from the obvious fact $\left\|D w_{0}(z)\right\|=\max _{\theta}\left|\partial_{\theta} w_{0}(z)\right|$ that

$$
\left\|D w_{0}(z)\right\| \leq L_{1} \text {. }
$$

On the other hand, if $\left\|D w_{0}(z)\right\| \leq L_{1}$, then the mean-value theorem of differentials leads to

$$
\left|w_{0}\left(z_{1}\right)-w_{0}\left(z_{2}\right)\right| \leq L_{1}\left|z_{1}-z_{2}\right| .
$$

Hence the claim is true.

Since we have proved that $\left\|D w_{0}(z)\right\|$ is unbounded, we see from Claim 6.1 that $w_{0}$ is not Lipschitz continuous in $\mathbb{D}$, which shows that the second assertion in the example holds too. The third assertion follows from [3, p. 317] as the construction of $w_{0}$ in $\mathbb{S}^{1}$ coincides with the one in [3].

Now, we construct an example to show that there exists a hyperbolic harmonic mapping $u$, which satisfies the conditions of Theorem 1.3 but $u$ is not $K$-quasiregular. Recall that Example 1 of [3] shows that Lipschitz continuity of $\phi: \mathbb{S}^{n-1} \rightarrow \mathbb{R}^{n}$ does not imply Lipschitz continuity of its harmonic extension $u=P[\phi]: \mathbb{B}^{n} \rightarrow \mathbb{R}^{n}$, without the assumption that $u$ is quasiregular.

Example 6.2. For $n=4$, let $u(x)=\left(1-\frac{1}{3}|x|^{2}\right) x$ in $\mathbb{B}^{4}$. Then

(1) $u \in C^{2}\left(\mathbb{B}^{4}, \mathbb{R}^{4}\right) \cap C\left(\overline{\mathbb{B}^{4}}, \mathbb{R}^{4}\right)$ and $\Delta_{h} u=0$ in $\mathbb{B}^{4}$;

(2) $u=P_{h}[\phi]$, where $\phi(\xi)=\frac{2}{3} \xi$ and $\xi \in \mathbb{S}^{3}$;

(3) $u$ is not $K$-quasiregular for any $K \geq 1$;

(4) $u$ is Lipschitz continuous in $\mathbb{B}^{4}$;

(5) $\phi$ is Lipschitz continuous in $\mathbb{S}^{3}$.

Proof. Elementary calculations and Theorem B show that $\Delta_{h} u=0$ and $u$ satisfies the conditions (1) and (2).

For any $x=\left(x_{1}, \cdots, x_{4}\right) \in \mathbb{B}^{4}$, it follows from (2.1) that

$$
D u(x)=\left(\begin{array}{cccc}
1-\frac{1}{3}|x|^{2}-\frac{2}{3} x_{1}^{2} & -\frac{2}{3} x_{1} x_{2} & -\frac{2}{3} x_{1} x_{3} & -\frac{2}{3} x_{1} x_{4} \\
-\frac{2}{3} x_{1} x_{2} & 1-\frac{1}{3}|x|^{2}-\frac{2}{3} x_{2}^{2} & -\frac{2}{3} x_{2} x_{3} & -\frac{2}{3} x_{2} x_{4} \\
-\frac{2}{3} x_{1} x_{3} & -\frac{2}{3} x_{2} x_{3} & 1-\frac{1}{3}|x|^{2}-\frac{2}{3} x_{3}^{2} & -\frac{2}{3} x_{3} x_{4} \\
-\frac{2}{3} x_{1} x_{4} & -\frac{2}{3} x_{2} x_{4} & -\frac{2}{3} x_{3} x_{4} & 1-\frac{1}{3}|x|^{2}-\frac{2}{3} x_{4}^{2}
\end{array}\right) .
$$

By calculations, we see that the eigenvalues of $D u(x)^{T} \times D u(x)$ are

$$
\lambda_{1}^{2}=\left(1-|x|^{2}\right)^{2}, \quad \lambda_{2}^{2}=\lambda_{3}^{2}=\lambda_{4}^{2}=\frac{1}{9}\left(3-|x|^{2}\right)^{2} .
$$


Thus,

$$
J_{u}(x)=\frac{1}{27}\left(3-|x|^{2}\right)^{3}\left(1-|x|^{2}\right) \text { and }\|D u(x)\|=1-\frac{1}{3}|x|^{2},
$$

which implies that $u$ is not $K$-quasiregular in $\mathbb{B}^{4}$, and the statement (3) holds.

Further, for any $x, y \in \mathbb{B}^{4}$ and $\xi, \eta \in \mathbb{S}^{3}$, we have

$$
\begin{aligned}
|u(x)-u(y)| & \leq|x-y|+\left.\frac{1}{3}|| x\right|^{2} x-|y|^{2} y \mid \\
& \leq|x-y|+\frac{1}{3}|x|^{2} \cdot|x-y|+\frac{1}{3}\left(|x|^{2}-|y|^{2}\right)|y| \\
& \leq 2|x-y|,
\end{aligned}
$$

and $|\phi(\xi)-\phi(\eta)|=\frac{2}{3}|\xi-\eta|$. Thus, the statements (4) and (5) hold.

\section{ACKNOWLEDGEMENTS}

We thank Professor Manfred Stoll for useful suggestions to this paper.

\section{REFERENCES}

1. P. Ahern and W. Cohn, Weighted maximal functions and derivatives of invariant Poisson integrals of potentials, Pacific J. Math., 163 (1994), 1-16.

2. L. Ahlfors, Möbius transformations in several dimensions, Ordway Professorship Lectures in Mathematics. University of Minnesota, School of Mathematics, Minneapolis, Minn., 1981.

3. M. Arsenović, V. Kojić and M. Mateljević, On Lipschitz continuity of harmonic quasiregular maps on the unit ball in $\mathbb{R}^{n}$, Ann. Acad. Sci. Fenn. Math., 33 (2008), 315-318.

4. M. Arsenović and V. Manojlović, On the modulus of continuity of harmonic quasiregular mappings on the unit ball in $\mathbb{R}^{n}$, Filomat, 23 (2009), 199-201.

5. S. Axler, P. Bourdon and W. Ramey, Harmonic function theory, Springer-Verlag, New York, 1992.

6. A. Beardon, The geometry of discrete group, Springer-Verlag, New York, 1995.

7. J. Chen, P. Li, S. Sahoo and X. Wang, On the Lipschitz continuity of certain quasiregular mappings between smooth Jordan domains, submitted.

8. M. Chen and X. Chen, $\left(K, K^{\prime}\right)$-quasiconformal harmonic mappings of the upper half plane onto itself, Ann. Acad. Sci. Fenn. Math., 37 (2012), 265-276.

9. Sh. Chen and Zh. Su, Radial growth and Hardy-Littlewood-type theorems on hyperbolic harmonic functions, Filomat, 29 (2015), 361-370.

10. J. Clunie and T. Sheil-Small, Harmonic univalent functions, Ann. Acad. Sci. Fenn. Ser. A. I. 9 (1984), 3-25.

11. P. Duren, Harmonic mappings in the plane, Cambridge University Press, Cambridge, 2004.

12. K. Dyakonov, Equivalent norms on Lipschitz-type spaces of holomorphic functions, Acta Math., 178 (1997), 143-167.

13. K. Dyakonov, Holomorphic functions and quasiconformal mappings with smooth moduli, $A d v$. Math., 187 (2004), 146-172.

14. C. Graham, The Dirichlet problem for the Bergman Laplacian. I, Comm. Partial Differential Equations, 8 (1983), 433-476.

15. L. Hörmander, Notion of convexity, Progress in Mathematics, Vol. 127, Birkhäuser Boston Inc., Boston, MA, 1994.

16. D. Kalaj, On harmonic quasiconformal self-mappings of the unit ball, Ann. Acad. Sci. Fenn. Math., 33 (2008), 261-271.

17. D. Kalaj, Quasiconformal and harmonic mappings between Jordan domains, Math. Z., 260 (2008), 237-252. 
18. D. Kalaj, On the quasiconformal self-mappings of the unit ball satisfying the Poisson differential equation, Ann. Acad. Sci. Fenn. Math., 36 (2011), 177-194.

19. D. Kalaj and M. Mateljević, Inner estimate and quasiconformal harmonic maps between smooth domains, J. Anal. Math., 100 (2006), 117-132.

20. D. Kalaj and M. Mateljević, $\left(K, K^{\prime}\right)$-quasiconformal harmonic mappings, Potential Anal., 36 (2012), 117-135.

21. D. Kalaj and M. Pavlović, Boundary correspondence under harmonic quasiconformal diffeomorphisms of a half-plane, Ann. Acad. Sci. Fenn. Math., 30 (2005), 159-165.

22. D. Kalaj and M. Pavlović, On quasiconformal self-mappings of the unit disk satisfying Poisson's equation, Trans. Amer. Math. Soc., 363 (2011), 4043-4061.

23. D. Kalaj and D. Vujadinović, The solution operator of the inhomogeneous Dirichlet problem in the unit ball, Proc. Amer. Math. Soc., 144 (2016), 623-635.

24. V. Lappalainen, Liph-extension domains, Ann. Acad. Sci. Fenn. Math. Diss., 56, 1985.

25. P. Li, J. Chen and X. Wang, Quasiconformal solutions of Poisson equations, Bull. Aust. Math. Soc., 92 (2015), 420-428.

26. M. Pavlović, Boundary correspondence under harmonic quasiconformal homeomorphisms of the unit disk, Ann. Acad. Sci. Fenn. Math., 27 (2002), 365-372.

27. M. Pavlović, Introduction to function spaces on the disk, Posebna Izdanja 20. Matematički Institut SANU, Belgrade, 2004.

28. M. Pavlović, Lipschitz conditions on the modulus of a harmonic function, Rev. Mat. Iberoam, 23 (2007), 831-845.

29. E. Rainville, Special functions, Chelsea Publ. Comp., New York, 1971.

30. G. Ren and U. Kähler, Weighted Hölder continuity hyperbolic harmonic Bloch functions, $Z$. Angew. Math. Phys., 21 (2002), 599-610.

31. S. Rickman, Quasiregular mappings, Springer-Verlag, Berlin, 1993.

32. W. Rudin, Principles of mathematical analysis, third edition, McGraw-Hill Book Co., New York, 1976.

33. W. Rudin, Real and complex analysis, third edition, McGraw-Hill Book Co., New York, 1987.

34. M. Stoll, Harmonic function theory on real hyperbolic space. Lecture Notes (Preprint), 1999. http://www.math.sc.edu/people/faculty/stoll/hyperbolic.pdf.

35. M. Stoll, Harmonic and subharmonic function theory on the hyperbolic ball, Cambridge University Press, Cambridge, 2016.

36. M. Stoll, Weighted Dirichlet spaces of harmonic functions on the real hyperbolic ball, Complex Var. Elliptic Equ., 57 (2012), 63-89.

37. M. Vuorinen, Conformal geometry and quasiregular mappings (Monograph, 208 pp.), Lecture Notes in Math. Vol. 1319, Springer-Verlag, 1988.

38. K. Zhu, Spaces of holomorphic functions in the unit ball, Springer-Verlag, New York, 2005.

Jiaolong Chen, Department of Mathematics, Shantou University, Shantou, GuangDONG 515063, PeOple's Republic of ChinA

Email address: jiaolongchen@sina.com

Manzi Huang, Department of Mathematics, Hunan Normal University, Changsha, Hunan 410081, People's Republic of China

Email address: mzhuang79@163.com

Antti Rasila, Department of Mathematics and Systems Analysis, Aalto UniverSity, P. O. Box 11100, FI-00076 Aalto, Finland.

Email address: antti.rasila@iki.fi

Xiantao Wang, Department of Mathematics, Shantou University, Shantou, GuangDONG 515063, People's Republic of China

Email address: xtwang@stu.edu.cn 